\title{
Fermion masses and mixings and some phenomenological aspects of a 3-3-1 model with linear seesaw mechanism
}

\author{
A. E. Cárcamo Hernández, Nicolás A. Pérez-Julve, ${ }^{\dagger}$ and Yocelyne Hidalgo Velásquez \\ Universidad Técnica Federico Santa María and Centro Científico-Tecnológico de Valparaíso, \\ Casilla 110-V, Valparaíso, Chile
}

(Received 3 September 2019; published 26 November 2019)

\begin{abstract}
We propose a viable theory based on the $S U(3)_{C} \times S U(3)_{L} \times U(1)_{X}$ gauge group supplemented by the $S_{4}$ discrete group together with other various symmetries, whose spontaneous breaking gives rise to the current Standard Model (SM) fermion mass and mixing hierarchy. In the proposed theory the small light active neutrino masses are generated from a linear seesaw mechanism mediated by three Majorana neutrinos. The model is capable of reproducing the experimental values of the physical observables of both quark and lepton sectors. Our model is predictive in the quark sector having 9 effective parameters that allow to successfully reproduce the four Cabbibo-Kobayashi-Maskawa parameters and the six SM quark masses. In the SM quark sector, there is particular scenario, motivated by naturalness arguments, which allows a good fit for its ten observables, with only six effective parameters. We also study the single heavy scalar production via gluon fusion mechanism at a proton-proton collider. Our model is also consistent with the experimental constraints arising from the Higgs diphoton decay rate.
\end{abstract}

DOI: 10.1103/PhysRevD.100.095025

\section{INTRODUCTION}

Although the Standard Model (SM) is a very well established quantum field theory highly consistent with the experimental data, it has several unexplained issues. For instance, the current pattern of SM fermion masses and mixing angles, the number of SM fermion families, the tiny values of active neutrino masses are some of the issues that do not find an explanation within the context of the SM. The SM fermion mass hierarchy is spanned over a range of 13 orders of magnitude from the light active neutrino mass scale up to the top quark mass. In addition, the experimental data shows that the quark mixing pattern is significantly different from the leptonic mixing one. The mixing angles of the quark sector are small, thus implying that the CabbiboKobayashi-Maskawa (CKM) quark mixing matrix is close to the identity matrix. On the other hand, two of the leptonic mixing angles are large and one is small, of the order of the Cabbibo angle, thus implying a Pontecorvo-MakiNakagawa-Sakata (PMNS) leptonic mixing matrix very different from the identity matrix. This is the so called

\footnotetext{
*antonio.carcamo@usm.cl †nicolasperezjulve@gmail.com

"yocehidalgov@gmail.com
}

Published by the American Physical Society under the terms of the Creative Commons Attribution 4.0 International license. Further distribution of this work must maintain attribution to the author(s) and the published article's title, journal citation, and DOI. Funded by SCOAP. flavor puzzle, which is not addressed by the SM and provides reason for considering models with augmented field content and extended symmetry groups added to explain the current SM fermion mass spectrum and mixing parameters.

Theories with an extended $S U(3)_{C} \times S U(3)_{L} \times U(1)_{X}$ gauge symmetry [1-49] (3-3-1 models) are used to explain the origin of the three family structure in the fermion sector, which is left unexplained in the SM. In these models, the chiral anomaly cancellation condition is fulfilled when there are equal number of $S U(3)_{L}$ fermionic triplets and antitriplets, which occurs when the number of fermion families is a multiple of three. In addition, when the chiral anomaly cancellation condition is combined with the asymptotic freedom in QCD, theories based on the $S U(3)_{C} \times S U(3)_{L} \times$ $U(1)_{X}$ gauge symmetry predict the existence of three fermion families. Furthermore, the large mass difference between the heaviest quark and the two lighter ones can be explained in 3-3-1 models due to the fact that the third family is treated under a different representation than the first and second ones.

Furthermore, the 3-3-1 models explain the quantization of the electric charge [50,51], have sources of $C P$ violation [52,53], have a natural Peccei-Quinn symmetry, which solves the strong- $C P$ problem [54-57], predict the limit $\sin \theta_{W}^{2}<\frac{1}{4}$, for the weak mixing parameter. Besides that, if one includes heavy sterile neutrinos in the fermionic spectrum of the 3-3-1 models, such theories will have cold dark matter candidates as weakly interacting massive particles (WIMPs) [58-61]. A concise review of WIMPs 
TABLE I. Scalar transformations under the $S U(3)_{C} \times S U(3)_{L} \times U(1)_{X} \times S_{4} \times Z_{6} \times Z_{12} \times Z_{16}$ group.

\begin{tabular}{lcccccccccccccccc}
\hline \hline & $\chi$ & $\eta$ & $\rho$ & $\sigma_{1}$ & $\sigma_{2}$ & $\xi$ & $\Delta$ & $\Theta$ & $\Phi$ & $\Xi$ & $\Omega$ & $\varphi$ & $\phi$ & $\zeta$ & $\Sigma$ & $S$ \\
\hline$S U(3)_{C}$ & $\mathbf{1}$ & $\mathbf{1}$ & $\mathbf{1}$ & $\mathbf{1}$ & $\mathbf{1}$ & $\mathbf{1}$ & $\mathbf{1}$ & $\mathbf{1}$ & $\mathbf{1}$ & $\mathbf{1}$ & $\mathbf{1}$ & $\mathbf{1}$ & $\mathbf{1}$ & $\mathbf{1}$ & $\mathbf{1}$ & $\mathbf{1}$ \\
$S U(3)_{L}$ & $\mathbf{3}$ & $\mathbf{3}$ & $\mathbf{3}$ & $\mathbf{1}$ & $\mathbf{1}$ & $\mathbf{1}$ & $\mathbf{1}$ & $\mathbf{1}$ & $\mathbf{1}$ & $\mathbf{1}$ & $\mathbf{1}$ & $\mathbf{1}$ & $\mathbf{1}$ & $\mathbf{1}$ & $\mathbf{1}$ & $\mathbf{1}$ \\
$U(1)_{X}$ & $-\frac{1}{3}$ & $-\frac{1}{3}$ & $\frac{2}{3}$ & 0 & 0 & 0 & 0 & 0 & 0 & 0 & 0 & 0 & 0 & 0 & 0 & 0 \\
$S_{4}$ & $\mathbf{1}$ & $\mathbf{1}$ & $\mathbf{1}$ & $\mathbf{1}$ & $\mathbf{1}^{\prime}$ & $\mathbf{2}$ & $\mathbf{2}$ & $\mathbf{2}$ & $\mathbf{3}$ & $\mathbf{3}$ & $\mathbf{3}$ & $\mathbf{3}$ & $\mathbf{3}^{\prime}$ & $\mathbf{3}^{\prime}$ & $\mathbf{3}^{\prime}$ & $\mathbf{3}^{\prime}$ \\
$Z_{6}$ & 0 & 0 & 0 & 0 & 0 & 0 & 0 & 0 & 2 & 2 & 2 & 1 & 1 & 0 & 0 & 0 \\
$Z_{12}$ & 0 & 0 & 0 & -3 & 1 & -5 & -4 & -2 & 1 & 0 & 1 & 1 & 1 & -2 & 0 & 0 \\
$Z_{16}$ & 0 & 0 & 0 & -1 & -1 & -2 & -1 & -1 & 0 & 1 & 0 & 0 & 0 & 0 & 0 & 1 \\
\hline \hline
\end{tabular}

in 3-3-1 electroweak gauge models is provided in Ref. [62]. Finally, if one considers 3-3-1 electroweak gauge models with three right handed Majorana neutrinos and without exotic charges, one can implement a low scale linear or inverse seesaw mechanism, useful for generating the tiny active neutrinos masses.

In this work, motivated by the aforementioned considerations, we propose an extension of the 3-3-1 model with right handed Majorana neutrinos, where the scalar spectrum is enlarged by the inclusion of several gauge singlet scalars. Our theoretical construction successfully explains the current SM fermion mass spectrum and fermionic mixing parameters. In the proposed model, the $S U(3)_{C} \times$ $S U(3)_{L} \times U(1)_{X}$ gauge symmetry is supplemented by the $S_{4}$ family symmetry and other auxiliary cyclic symmetries, whose spontaneous breaking produces the current SM fermion mass spectrum and mixing parameters. In the proposed model, the masses for the Standard Model charged fermions lighter than the top quark are produced by a Froggatt-Nielsen mechanism and the tiny masses for the light active neutrinos are generated by a linear seesaw mechanism. We employ the $S_{4}$ family symmetry because it is the smallest non-Abelian group having a doublet, triplet, and singlet irreducible representations, thus permitting us to accommodate the three fermion families of the SM. It is worth mentioning that the $S_{4}$ discrete group [10,63-84] has been shown to provide a nice description for the observed pattern of SM fermion masses and mixing angles.

The layout of the remainder of the paper is as follows. In Sec. II A we describe the proposed model, its symmetries, particle content and Yukawa interactions. The gauge sector of the model is described in Sec. II B, whereas its low energy scalar potential is presented in Sec. II C. In Sec. III we discuss the implications of our model in quark masses and mixings. In Sec. IV, we present our results on lepton masses and mixing. The consequences of our model in the Higgs diphoton decay rate are discussed in Sec. V. The production of the heavy $H_{1}$ scalar at proton-proton collider is discussed in Sec. VI. We conclude in Sec. VII. Appendix A provides a description of the $S_{4}$ discrete group. Appendices $\mathrm{B}$ and $\mathrm{C}$ present a discussion of the scalar potentials for a $S_{4}$ scalar doublet and $S_{4}$ triplet, respectively.

\section{THE MODEL}

\section{A. Particle spectrum and symmetries}

We propose an extension of the 3-3-1 model with right handed Majorana neutrinos, where the $S U(3)_{C} \times S U(3)_{L} \times$ $U(1)_{X}$ gauge symmetry is augmented by the $S_{4} \times Z_{6} \times$ $Z_{12} \times Z_{16}$ discrete group and the scalar spectrum is enlarged by considering gauge singlet scalars, which are added in order to generate viable textures for the fermion sector that successfully explain the current pattern of SM fermion masses and mixing angles. The scalar and fermionic content with their assignments under the $S U(3)_{C} \times$ $S U(3)_{L} \times U(1)_{X} \times S_{4} \times Z_{6} \times Z_{12} \times Z_{16}$ group are given in Tables I and II, respectively. The dimensions of the $S U(3)_{C}$, $S U(3)_{L}$ and $S_{4}$ representations shown in Tables I and II, are described by numbers in boldface and the additive notation is used to specify the $U(1)_{X}$ and $Z_{N}$ charges. We choose the $S_{4}$ symmetry since it is the smallest non-Abelian group having doublet, triplet, and singlet irreducible representations, thus allowing us to naturally accommodate the

TABLE II. Fermion transformations under the $S U(3)_{C} \times S U(3)_{L} \times U(1)_{X} \times S_{4} \times Z_{6} \times Z_{12} \times Z_{16}$ group.

\begin{tabular}{lccccccccccccccccc}
\hline \hline & $q_{1 L}$ & $q_{2 L}$ & $q_{3 L}$ & $u_{1 R}$ & $u_{2 R}$ & $u_{3 R}$ & $d_{1 R}$ & $d_{R}$ & $t_{R}^{\prime}$ & $j_{1 R}$ & $j_{2 R}$ & $L_{L}$ & $e_{1 R}$ & $e_{2 R}$ & $e_{3 R}$ & $N_{1 R}$ & $N_{R}$ \\
\hline$S U(3)_{C}$ & $\mathbf{3}$ & $\mathbf{3}$ & $\mathbf{3}$ & $\mathbf{3}$ & $\mathbf{3}$ & $\mathbf{3}$ & $\mathbf{3}$ & $\mathbf{3}$ & $\mathbf{3}$ & $\mathbf{3}$ & $\mathbf{3}$ & $\mathbf{1}$ & $\mathbf{1}$ & $\mathbf{1}$ & $\mathbf{1}$ & $\mathbf{1}$ & $\mathbf{1}$ \\
$S U(3)_{L}$ & $\mathbf{3}^{*}$ & $\mathbf{3}^{*}$ & $\mathbf{3}$ & $\mathbf{1}$ & $\mathbf{1}$ & $\mathbf{1}$ & $\mathbf{1}$ & $\mathbf{1}$ & $\mathbf{1}$ & $\mathbf{1}$ & $\mathbf{1}$ & $\mathbf{3}$ & $\mathbf{1}$ & $\mathbf{1}$ & $\mathbf{1}$ & $\mathbf{1}$ & $\mathbf{1}$ \\
$U(1)_{X}$ & 0 & 0 & $\frac{1}{3}$ & $\frac{2}{3}$ & $\frac{2}{3}$ & $\frac{2}{3}$ & $-\frac{1}{3}$ & $-\frac{1}{3}$ & $\frac{2}{3}$ & $-\frac{1}{3}$ & $-\frac{1}{3}$ & $-\frac{1}{3}$ & -1 & -1 & -1 & 0 & 0 \\
$S_{4}$ & $\mathbf{1}$ & $\mathbf{1}$ & $\mathbf{1}$ & $\mathbf{1}$ & $\mathbf{1}$ & $\mathbf{1}$ & $\mathbf{1}^{\prime}$ & $\mathbf{2}$ & $\mathbf{1}$ & $\mathbf{1}$ & $\mathbf{1}$ & $\mathbf{3}$ & $\mathbf{1}^{\prime}$ & $\mathbf{1}$ & $\mathbf{1}$ & $\mathbf{1}^{\prime}$ & $\mathbf{2}$ \\
$Z_{6}$ & 0 & 0 & 0 & 0 & 0 & 0 & 0 & 0 & 0 & 0 & 0 & 0 & -1 & -1 & -2 & 0 & 0 \\
$Z_{12}$ & 0 & 0 & 0 & 4 & 0 & 0 & 5 & 0 & 0 & 0 & 0 & -1 & 2 & -6 & -4 & -1 & -1 \\
$Z_{16}$ & -4 & -2 & 0 & 4 & 2 & 0 & 3 & 3 & 0 & -4 & -2 & 0 & 8 & 4 & 2 & 0 & -1 \\
\hline \hline
\end{tabular}


three families of the SM left handed leptonic fields into a $S_{4}$ triplet, the three gauge singlet right handed Majorana neutrinos into one $S_{4}$ singlet and one $S_{4}$ doublet, the three right handed SM down type quarks into a $S_{4}$ singlet and a $S_{4}$ doublet, and the remaining fermionic fields as $S_{4}$ singlets. In addition, the $S_{4}, Z_{6}$, and $Z_{12}$ symmetries shape the textures of the SM fermion mass matrices thus yielding a reduction of the model parameters, especially in the SM quark sector. In addition, the $Z_{6}$ and $Z_{12}$ symmetries separate the $S_{4}$ scalar triplets $(\Phi, \Xi, \Omega, \varphi, \phi)$ participating in the charged lepton Yukawa interactions, from the ones $(\zeta, \Sigma, S)$ appearing in the neutrino Yukawa terms. Moreover, the $Z_{6}$ symmetry allows to distinguish the $S_{4}$ scalar triplets $\Phi, \Xi, \Omega$ generating the third column of the SM charged lepton mass matrix from the ones $\varphi$ and $\phi$ that give rise to the second and the first column of the SM charged lepton mass matrix, respectively. Consequently, the $Z_{6}$ symmetry selects the allowed entries of the SM charged lepton mass matrix, thus allowing to reduce the number of lepton sector model parameters. Furthermore, the $Z_{12}$ symmetry distinguishes the $S_{4}$ scalar triplet $\zeta$ participating in the Dirac Yukawa interactions, from the ones $\Sigma$ and $S$ appearing in the remaining neutrino Yukawa terms. Besides that, the $Z_{12}$ symmetry also distinguishes the different $S_{4}$ scalar doublets $\xi, \Delta$, and $\Theta$ that contribute to the first, second and third rows of the SM down type quark mass matrix, respectively, thus allowing to obtain a predictive texture for the down type quark sector that generates the SM down quark masses, the Cabbibo mixing as well as the mixing between the second and third quark families. Furthermore, the $Z_{12}$ symmetry also determines the allowed entries of the SM up type quark mass matrix. It is worth mentioning that due to the $Z_{12}$ charge assignments, the only nonvanishing entries of the SM up type quark mass matrix are the diagonal ones as well as the 13 entry, needed to generate the SM up quark masses as well as the quark mixing angle in the 13 plane and the quark $C P$ violating phase, respectively. The quark mixing angle in the 13 plane and the quark $C P$ violating phase only arise from the up type quark sector. The $Z_{16}$ symmetry shapes the hierarchical structure of the SM fermion mass matrices crucial to yield the observed SM fermion mass and mixing pattern. We remark that $Z_{16}$ is the smallest discrete symmetry permitting to build the Yukawa terms $\left(\bar{l}_{L} \rho \Pi\right)_{1} e_{1 R} \frac{\sigma_{2}^{8}}{\Lambda^{9}}(\Pi=\Phi, \Xi, \Omega)$ and $\bar{q}_{1 L} \rho^{*} u_{1 R} \frac{\sigma_{2}^{8}}{\Lambda^{8}}$, required to provide a natural explanation for the small values of the electron and up quark masses, which are $\lambda^{9} \frac{v}{\sqrt{2}}$ and $\lambda^{8} \frac{v}{\sqrt{2}}$ times a $\mathcal{O}(1)$ coupling, respectively, where $\lambda=0.225$ is one of the Wolfenstein parameters. In our model, the masses of the Standard Model charged fermions lighter than the top quark arise from a Froggatt-Nielsen mechanism [85], triggered by nonrenormalizable Yukawa interactions invariant under the different discrete group factors. Thus, the current pattern of SM fermion masses and mixing angles arises from the $S_{4} \times Z_{6} \times Z_{12} \times Z_{16}$ spontaneous symmetry breaking. The masses of the light active neutrinos are generated from a linear seesaw mechanism [86-90,90-94], which can be implemented in our model because the third component of the $S U(3)_{L}$ leptonic triplet is electrically neutral and the fermionic spectrum includes three right handed Majorana neutrinos. In addition, the non-SM fermions in our model do not have exotic electric charges. Consequently, the electric charge is defined as:

$$
Q=T_{3}+\beta T_{8}+X I=T_{3}-\frac{1}{\sqrt{3}} T_{8}+X I,
$$

with $I=\operatorname{diag}(1,1,1), \quad T_{3}=\frac{1}{2} \operatorname{diag}(1,-1,0)$ and $T_{8}=$ $\left(\frac{1}{2 \sqrt{3}}\right) \operatorname{diag}(1,1,-2)$ for a $S U(3)_{L}$ triplet. In our model the full symmetry $\mathcal{G}$ experiences the three-step spontaneous breaking pattern:

$$
\begin{aligned}
\mathcal{G}= & S U(3)_{C} \times S U(3)_{L} \times U(1)_{X} \times S_{4} \times Z_{6} \times Z_{12} \times Z_{16} \stackrel{\Lambda_{\text {int }}}{\rightarrow} \\
& S U(3)_{C} \times S U(3)_{L} \times U(1)_{X} \stackrel{v_{x}}{\rightarrow} \\
& S U(3)_{C} \times S U(2)_{L} \times U(1)_{Y} \stackrel{v_{n}, v_{\rho}}{\rightarrow} \\
& S U(3)_{C} \times U(1)_{Q},
\end{aligned}
$$

where the different symmetry breaking scales satisfy $\Lambda_{\text {int }} \gg v_{\chi} \gg v_{\eta}, v_{\rho}$, with $\sqrt{v_{\eta}^{2}+v_{\rho}^{2}}=246 \mathrm{GeV}$. The first step of spontaneous symmetry breaking is produced by all gauge singlet scalar fields (excepting $\zeta$ ), charged under the discrete symmetries, assumed to acquire vacuum expectation values (VEVs) at a very large energy scale $\Lambda_{\text {int }} \gg v_{\chi} \sim \mathcal{O}(10) \mathrm{TeV}$. The second step of spontaneous symmetry breaking is caused by the $S U(3)_{L}$ scalar triplet $\chi$, whose third component acquires a $10 \mathrm{TeV}$ scale vacuum expectation value $(\mathrm{VEV})$ that breaks the $S U(3)_{L} \times U(1)_{X}$ gauge symmetry, thus providing masses for the exotic fermions, non-Standard Model gauge bosons and the heavy $C P$ even neutral scalar state of $\chi$. We further assume that the $S_{4}$ triplet gauge singlet scalar $\zeta$ acquires a VEVs at the same scale of $v_{\chi}$. Finally, the remaining two $S U(3)_{L}$ scalar triplets $\eta$ and $\rho$, whose first and second components, respectively, get VEVs at the Fermi scale, thus producing the masses for the SM particles and for the physical neutral scalar states arising from those scalar triplets. Here we are considering that the $S U(3)_{L} \times U(1)_{X}$ gauge symmetry is spontaneously broken at a scale of about $10 \mathrm{TeV}$ in order to comply with collider constraints [95] as well as with the constraints arising from the experimental data on $K, D$, and $B$ meson mixings [96] and from the $B_{s, d} \rightarrow \mu^{+} \mu^{-}$and $B_{d} \rightarrow$ $K^{*}(K) \mu^{+} \mu^{-}$decays [9,97-100].

The $S U(3)_{L}$ triplet scalars $\chi, \eta$, and $\rho$ can be represented as: 


$$
\begin{array}{lc}
\chi=\left(\begin{array}{c}
\chi_{1}^{0} \\
\chi_{2}^{-} \\
\frac{1}{\sqrt{2}}\left(v_{\chi}+\xi_{\chi} \pm i \zeta_{\chi}\right)
\end{array}\right), & q_{n L}=\left(\begin{array}{c}
d_{n} \\
-u_{n} \\
j_{n}
\end{array}\right)_{L}, \quad q_{3 L}=\left(\begin{array}{c}
u_{3} \\
d_{3} \\
t^{\prime}
\end{array}\right)_{L} \\
\eta=\left(\begin{array}{c}
\frac{1}{\sqrt{2}}\left(v_{\eta}+\xi_{\eta} \pm i \zeta_{\eta}\right) \\
\eta_{2}^{-} \\
\eta_{3}^{0}
\end{array}\right), & L_{i L}=\left(\begin{array}{c}
\nu_{i} \\
e_{i} \\
\nu_{i}^{c}
\end{array}\right)_{L}, \quad n=1,2, \quad i=1,2,3 .
\end{array}
$$

$$
\rho=\left(\begin{array}{c}
\rho_{1}^{+} \\
\frac{1}{\sqrt{2}}\left(v_{\rho}+\xi_{\rho} \pm i \zeta_{\rho}\right) \\
\rho_{3}^{+}
\end{array}\right) .
$$

The $S U(3)_{L}$ fermionic antitriplets and triplets are expressed as:

$$
\begin{aligned}
-\mathcal{L}_{Y}^{(q)}= & y^{\left(t^{\prime}\right)} \bar{q}_{3 L} \chi t_{R}^{\prime}+y_{33}^{(u)} \bar{q}_{3 L} \eta u_{3 R}+y_{13}^{(u)} \bar{q}_{1 L} \rho^{*} u_{3 R} \frac{\sigma_{1}^{4}}{\Lambda^{4}}+y_{22}^{(u)} \bar{q}_{2 L} \rho^{*} u_{2 R} \frac{\sigma_{1}^{4}}{\Lambda^{4}}+y_{11}^{(u)} \bar{q}_{1 L} \rho^{*} u_{1 R} \frac{\sigma_{2}^{8}}{\Lambda^{8}} \\
& +y_{1}^{(j)} \bar{q}_{1 L} \chi^{*} j_{1 R}+y_{2}^{(j)} \bar{q}_{2 L} \chi^{*} j_{2 R}+y_{3}^{(d)} \bar{q}_{3 L} \rho\left(\Theta d_{R}\right)_{1} \frac{\sigma_{2}^{2}}{\Lambda^{3}}+y_{2}^{(d)} \bar{q}_{2 L} \eta^{*}\left(\Delta d_{R}\right)_{1} \frac{\sigma_{2}^{4}}{\Lambda^{5}}+y_{4}^{(d)} \bar{q}_{1 L} \eta^{*}\left(\xi d_{R}\right)_{1} \frac{\sigma_{2}^{5}}{\Lambda^{6}} \\
& +y_{1}^{(d)} \bar{q}_{1 L} \eta^{*} d_{1 R} \frac{\sigma_{2}^{7}}{\Lambda^{7}}+\text { H.c., } \\
-\mathcal{L}_{Y}^{(l)}= & x_{1}^{(L)}\left(\bar{L}_{L} \rho \phi\right)_{1} e_{1 R} \frac{\sigma_{2}^{8}}{\Lambda^{9}}+y_{1}^{(L)}\left(\bar{L}_{L} \rho \varphi\right)_{1} e_{2 R} \frac{\sigma_{2}^{4}}{\Lambda^{5}}+y_{2}^{(L)}\left(\bar{L}_{L} \rho \varphi\right)_{1} e_{2 R} \frac{\sigma_{2}^{4}}{\Lambda^{5}} \\
& +z_{1}^{(L)}\left(\bar{L}_{L} \rho \Phi\right)_{1} e_{3 R} \frac{\sigma_{2}^{2}}{\Lambda^{3}}+z_{2}^{(L)}\left(\bar{L}_{L} \rho \Xi\right)_{1} e_{3 R} \frac{\sigma_{2}^{3}}{\Lambda^{4}}+z_{3}^{(L)}\left(\bar{L}_{L} \rho \Omega\right)_{1} e_{3 R} \frac{\sigma_{2}^{2}}{\Lambda^{3}}+y_{\rho} \varepsilon_{a b c}\left(\bar{L}_{L}^{a}\left(L_{L}^{C}\right)^{b}\right)_{3}\left(\rho^{*}\right)^{c} \frac{\zeta}{\Lambda} \\
& +y_{1 \chi}^{(L)}\left(\bar{L}_{L} \Sigma\right)_{1} \chi N_{1 R} \frac{1}{\Lambda}+y_{2 \chi}^{(L)} \bar{L}_{L} \chi\left(S N_{R}\right)_{3} \frac{1}{\Lambda}+y_{1 \eta}^{(L)}\left(\bar{L}_{L} \Sigma\right)_{1^{\prime}} \eta N_{1 R} \frac{1}{\Lambda}+y_{2 \eta}^{(L)} \bar{L}_{L} \eta\left(S N_{R}\right)_{3} \frac{1}{\Lambda}+\text { H.c. }
\end{aligned}
$$

As shown in detail in Appendices B and C, the following VEV configurations for the $S_{4}$ scalar doublets and $S_{4}$ scalar triplets are in accordance with the minimization conditions of the scalar potential:

$$
\begin{aligned}
&\langle\xi\rangle=v_{\xi}(0,-1), \quad\langle\Delta\rangle=\frac{v_{\Delta}}{\sqrt{5}}(2,1), \quad\langle\Theta\rangle=v_{\Theta}(1,0), \quad\langle\Phi\rangle=v_{\Phi}(1,0,0), \quad\langle\Xi\rangle=\frac{v_{\Xi}}{\sqrt{2}}(0,1,0), \\
&\langle\Omega\rangle=\frac{v_{\Omega}}{\sqrt{2}}(0,0,1), \quad\langle\varphi\rangle=\frac{v_{\varphi}}{\sqrt{1+r^{2}}}(1,0, r), \quad\langle\phi\rangle=v_{\phi}(1,0,0), \\
&\langle\zeta\rangle=\frac{v_{\zeta}}{\sqrt{1+c^{2}}}(1,0, c), \quad\langle\Sigma\rangle=\frac{v_{\Sigma}}{\sqrt{3}}(1,-1,1), \quad\langle S\rangle=\frac{v_{S}}{\sqrt{3}}(1,1,1) .
\end{aligned}
$$

Given that the spontaneous $S_{4} \times Z_{6} \times Z_{12} \times Z_{16}$ symmetry breaking gives rise to the observed hierarchy of SM charged fermion masses and quark mixing parameters, the vacuum expectation values of the gauge singlet scalars can be expressed in terms of the Wolfenstein parameter $\lambda=0.225$ and the model cutoff $\Lambda$, in the following way:

$$
v_{\eta} \sim v_{\rho} \ll v_{\zeta} \sim v_{\chi} \ll v_{\Delta} \sim v_{\Phi} \sim v_{\Xi} \sim v_{\Omega} \sim v_{\xi} \sim v_{\Theta} \sim v_{\Sigma} \sim v_{S} \sim v_{\sigma_{n}} \sim v_{\varphi} \sim v_{\phi} \sim \Lambda_{\text {int }}=\lambda \Lambda, \quad n=1,2 .
$$

where the model cutoff $\Lambda$ can be interpreted as the scale of the UV completion of the model, e.g., the masses of Froggatt-Nielsen messenger fields. 
TABLE III. Physical gauge bosons and their masses.

\begin{tabular}{lc}
\hline \hline Gauge boson & Square mass \\
\hline$W^{ \pm}$ & $\frac{1}{4} g^{2}\left(v_{\eta}^{2}+v_{\rho}^{2}\right)$ \\
$W^{\prime \pm}$ & $\frac{1}{4} g^{2}\left(v_{\chi}^{2}+v_{\rho}^{2}\right)$ \\
$\gamma$ & 0 \\
$Z$ & $\frac{1}{9}\left(\Xi_{1}-\Xi_{2}\right)$ \\
$Z^{\prime}$ & $\frac{1}{9}\left(\Xi_{1}+\Xi_{2}\right)$ \\
$K^{0}, \bar{K}^{0}$ & $\frac{g^{2}}{8}\left(v_{\chi}^{2}+v_{\eta}^{2}\right)$ \\
\hline \hline
\end{tabular}

\section{B. The gauge sector}

Using $\beta=-1 / \sqrt{3}$, the gauge bosons related with $S U(3)_{L}$ are:

$$
\begin{aligned}
\mathbf{W}_{\mu} & =W_{\mu}^{\alpha} G_{\alpha} \\
& =\frac{1}{2}\left(\begin{array}{ccc}
W_{\mu}^{3}+\frac{1}{\sqrt{3}} W_{\mu}^{8} & \sqrt{2} W_{\mu}^{+} & \sqrt{2} K_{\mu}^{0} \\
\sqrt{2} W_{\mu}^{-} & -W_{\mu}^{3}+W_{\mu}^{8} & \sqrt{2} K_{\mu}^{-} \\
\sqrt{2} \bar{K}_{\mu}^{0} & \sqrt{2} K_{\mu}^{+} & -\frac{2}{\sqrt{3}} W_{\mu}^{8}
\end{array}\right)
\end{aligned}
$$

where $G_{\alpha}(\alpha=1, \cdots, 8)$ are the Gell-Mann matrices. The representation of the gauge field related to the $U(1)_{X}$ gauge symmetry has $Q_{B}=0$ charge and is given by:

$$
\mathbf{B}_{\mu}=\mathbf{I}_{3 \times 3} B_{\mu}
$$

In this model three gauge fields with no electric charge are combined to form the photon as well as the $Z$ and $Z^{\prime}$ gauge bosons. Moreover, there are two gauge fields with electric

charge $\pm 1\left(W^{ \pm}\right)$and four non-SM gauge bosons $W^{\prime \pm}$, $\bar{K}^{0}, K^{0}$.

The covariant derivative in 3-3-1 models reads:

$$
D_{\mu}=\partial_{\mu}+i g W_{\mu}^{\alpha} G_{\alpha}+i g^{\prime} X_{\Phi} B_{\mu}
$$

Replacing Eq. (2.11) in the scalar kinetic interactions gives rise to the gauge boson mass terms as well as to the interactions between the scalar and gauge bosons [101]:

$$
\begin{aligned}
\mathcal{L}_{\text {Kin }}= & \sum_{\Phi=\eta, \rho, \chi}\left(D^{\mu} \Phi\right)^{\dagger}\left(D_{\mu} \Phi\right) \\
= & \sum_{\Phi=\eta, \rho, \chi}[\overbrace{\left(\partial^{\mu} \Phi\right)^{\dagger}\left(D_{\mu} \Phi\right)+\left(D^{\mu} \Phi\right)^{\dagger}\left(\partial_{\mu} \Phi\right)}^{(1)} \\
& -\left(\partial^{\mu} \Phi\right)^{\dagger}\left(\partial_{\mu} \Phi\right) \\
& +\overbrace{\Phi^{\dagger}\left(g W^{\mu}+g^{\prime} X_{\Phi} B^{\mu}\right)^{\dagger}\left(g W^{\mu}+g^{\prime} X_{\Phi} B^{\mu}\right) \Phi}^{(2)}],
\end{aligned}
$$

where the terms denoted as (1) give rise to the interactions between the Goldstone and massive gauge bosons. On the other hand, the terms denoted as (2) produce the masses for the gauge bosons and the interactions between the gauge bosons and the physical scalar fields. The gauge boson squared mass matrices read:

$M_{\text {charged }}^{2}=\left(\begin{array}{cc}\frac{1}{8} g^{2} v_{\eta}^{2}+\frac{1}{8} g^{2} v_{\rho}^{2} & 0 \\ 0 & \frac{1}{8} g^{2} v_{\rho}^{2}+\frac{1}{8} g^{2} v_{\chi}^{2}\end{array}\right)$

$$
M_{\text {neutral }}^{2}=\left(\begin{array}{cccc}
\frac{1}{4} g^{2}\left(v_{\eta}^{2}+v_{\rho}^{2}\right) & \frac{g^{2} v_{\eta}^{2}}{4 \sqrt{3}}-\frac{g^{2} v_{\rho}^{2}}{4 \sqrt{3}} & -\frac{1}{6} g g^{\prime} v_{\eta}^{2}-\frac{1}{3} g v_{\rho}^{2} g^{\prime} & 0 \\
\frac{g^{2} v_{\eta}^{2}}{4 \sqrt{3}}-\frac{g^{2} v_{\rho}^{2}}{4 \sqrt{3}} & \frac{1}{12} g^{2} v_{\eta}^{2}+\frac{1}{12} g^{2} v_{\rho}^{2}+\frac{1}{3} g^{2} v_{\chi}^{2} & -\frac{g g^{\prime} v_{\eta}^{2}}{6 \sqrt{3}}+\frac{g v_{\rho}^{2} g^{\prime}}{3 \sqrt{3}}+\frac{g v_{\chi}^{2} g^{\prime}}{3 \sqrt{3}} & 0 \\
-\frac{1}{6} g g^{\prime} v_{\eta}^{2}-\frac{1}{3} g v_{\rho}^{2} g^{\prime} & -\frac{g g^{\prime} v_{\eta}^{2}}{6 \sqrt{3}}+\frac{g v_{\rho}^{2} g^{\prime}}{3 \sqrt{3}}+\frac{g v_{\chi}^{2} g^{\prime}}{3 \sqrt{3}} & \frac{1}{9} v_{\eta}^{2}\left(g^{\prime}\right)^{2}+\frac{4}{9} v_{\rho}^{2}\left(g^{\prime}\right)^{2}+\frac{1}{9} v_{\chi}^{2}\left(g^{\prime}\right)^{2} & 0 \\
0 & 0 & 0 & \frac{1}{8} g^{2}\left(v_{\eta}^{2}+v_{\chi}^{2}\right)
\end{array}\right)
$$

The physical gauge bosons and their masses are shown in Table III:

$$
\begin{aligned}
& \Xi_{1}=3 g^{2}\left(v_{\eta}^{2}+v_{\rho}^{2}+v_{\chi}^{2}\right)+\left(g^{\prime}\right)^{2}\left(v_{\eta}^{2}+4 v_{\rho}^{2}+v_{\chi}^{2}\right) \\
& \Xi_{2}=\sqrt{\left(3 g^{2}\left(v_{\eta}^{2}+v_{\rho}^{2}+v_{\chi}^{2}\right)+\left(g^{\prime}\right)^{2}\left(v_{\eta}^{2}+4 v_{\rho}^{2}+v_{\chi}^{2}\right)\right)^{2}-9 g^{2}\left(3 g^{2}+4\left(g^{\prime}\right)^{2}\right)\left(v_{\eta}^{2}\left(v_{\rho}^{2}+v_{\chi}^{2}\right)+v_{\rho}^{2} v_{\chi}^{2}\right)}
\end{aligned}
$$

with $v_{\eta} \simeq 173.948 \mathrm{GeV}, v_{\rho} \simeq 173.948 \mathrm{GeV}$, and $v_{\chi} \simeq 10 \mathrm{TeV}$. Consequently, for these values we find that the heavy gauge boson masses are $M_{W^{\prime}} \approx 3.2 \mathrm{TeV}, M_{Z^{\prime}} \approx 6.3 \mathrm{TeV}$. Notice that the obtained value of $M_{Z^{\prime}} \approx 6.3 \mathrm{TeV}$ is consistent with the lower bound of $4 \mathrm{TeV}$ on the $Z^{\prime}$ gauge boson mass imposed by the experimental data on the $K, D$ and $B$ meson mixings [96].
In what follows we briefly comment about the LHC signals of a $Z^{\prime}$ gauge boson. The heavy $Z^{\prime}$ gauge boson is mainly produced via Drell-Yan mechanism and its corresponding production cross section has been found to range from $85 \mathrm{fb}$ up to $10 \mathrm{fb}$ for $Z^{\prime}$ gauge boson masses between $4 \mathrm{TeV}$ and $5 \mathrm{TeV}$ and LHC center of mass energy $\sqrt{S}=$ $13 \mathrm{TeV}$ [102]. Such $Z^{\prime}$ gauge boson after being produced 
will decay into pair of SM particles, with dominant decay mode into quark-antiquark pairs as shown in detail in Refs. $[9,103]$. Comprehensive studies of the two body decays of the $Z^{\prime}$ gauge boson in 3-3-1 models are performed in Refs. $[9,103]$, where it has been shown that the branching ratios of the $Z^{\prime}$ decays into a lepton pair are of the order of $10^{-2}$, thus yielding a total LHC cross section of about $1 \mathrm{fb}$ for the $p p \rightarrow Z^{\prime} \rightarrow l^{+} l^{-}$resonant production at $\sqrt{S}=13 \mathrm{TeV}$ and $M_{Z^{\prime}}=4 \mathrm{TeV}$, which is below its corresponding lower experimental bound resulting from LHC searches [104]. Finally, as pointed out in Ref. [102], at the proposed energy upgrade of the LHC with $\sqrt{S}=28 \mathrm{TeV}$, the LHC production cross section for the $p p \rightarrow Z^{\prime} \rightarrow l^{+} l^{-}$resonant production will be of the order of $10^{-2}$, at $M_{Z^{\prime}}=4 \mathrm{TeV}$, which falls in the order of magnitude of its corresponding experimental lower bound resulting from LHC searches.

\section{The low energy scalar potential and scalar mass spectrum}

In our model, the renormalizable low energy scalar potential reads:
TABLE IV. Physical scalar fields with their masses.

\begin{tabular}{ll}
\hline \hline Scalars & \multicolumn{1}{c}{ Masses } \\
\hline$G_{1}^{0}=-S_{\alpha} \zeta_{\chi}+C_{\alpha} \zeta_{\eta}$ & $M_{G_{1}^{0}}^{2}=0$ \\
$A^{0}=C_{\beta} \zeta_{\rho}+S_{\beta} \zeta_{\eta}$ & $M_{A^{0}}=\frac{f}{\sqrt{2}} v_{\chi}\left(\frac{v_{\rho}}{v_{\eta}}+\frac{v_{\eta}}{v_{\rho}}\right)$ \\
$G_{2}^{0}=-C_{\gamma} \zeta_{\chi}+S_{\gamma} \zeta_{\rho}$ & $M_{G_{2}^{0}}^{2}=0$ \\
$H_{1}^{0}=\xi_{\chi}$ & $M_{H_{1}^{0}}^{2}=\lambda_{1} v_{\chi}^{2}$ \\
$h^{0}=C_{\delta} \xi_{\rho}-S_{\delta} \xi_{\eta}$ & $M_{h^{0}}^{2}=\lambda_{3} v_{\eta}^{2}+\lambda_{2} v_{\rho}^{2}$ \\
$H_{2}^{0}=S_{\delta} \xi_{\rho}+C_{\delta} \xi_{\eta}$ & $M_{H_{2}^{0}}^{2}=\frac{f v_{\chi}}{\sqrt{2}}\left(\frac{v_{\eta}^{2}+v_{\rho}^{2}}{v_{\eta} v_{\rho}}\right)$ \\
$G_{3}^{0}=-C_{\alpha} \chi_{1}^{0}+S_{\alpha} \eta_{3}^{0}$ & $M_{G_{3}^{0}}^{2}=0$ \\
$\bar{G}_{3}^{0}=-C_{\alpha} \bar{\chi}_{1}^{0}+S_{\alpha} \bar{\eta}_{3}^{0}$ & $M_{\bar{G}_{3}^{0}}^{2}=0$ \\
$H_{3}^{0}=S_{\alpha} \chi_{1}^{0}+C_{\alpha} \eta_{3}^{0}$ & $M_{H_{3}^{0}}^{2}=\frac{\left(\sqrt{2} f v_{\rho}+\lambda_{\gamma} v_{\eta} v_{\chi}\right)\left(v_{\eta}^{2}+v_{\chi}^{2}\right)}{v_{\eta} v_{\chi}}$ \\
$\bar{H}_{3}^{0}=S_{\alpha} \bar{\chi}_{1}^{0}+C_{\alpha} \bar{\eta}_{3}^{0}$ & $M_{\bar{H}_{3}^{0}}^{2}=M_{H_{3}^{0}}^{2}$ \\
$G_{4}^{ \pm}=-C_{\gamma} \chi_{2}^{ \pm}+S_{\gamma} \rho_{3}^{ \pm}$ & $M_{G_{4}^{ \pm}}^{2}=0$ \\
$H_{4}^{ \pm}=S_{\gamma} \chi_{2}^{ \pm}+C_{\gamma} \rho_{3}^{ \pm}$ & $M_{H_{4}^{ \pm}}^{2}=\frac{\left(\sqrt{2} f v_{\eta}+\lambda_{8} v_{\rho} v_{\chi}\right)\left(v_{\rho}^{2}+v_{\chi}^{2}\right)}{v_{\rho} v_{\chi}}$ \\
$G_{5}^{ \pm}=-C_{\beta} \eta_{2}^{ \pm}+S_{\beta} \rho_{1}^{ \pm}$ & $M_{G_{5}^{ \pm}}^{2}=0$ \\
$H_{5}^{ \pm}=S_{\beta} \eta_{2}^{ \pm}+C_{\beta} \rho_{1}^{ \pm}$ & $M_{H_{5}^{ \pm}}^{2}=\frac{\left(\sqrt{2} f v_{\chi}+\lambda_{g} v_{\eta} v_{\rho}\right)\left(v_{\eta}^{2}+v_{\rho}^{2}\right)}{v_{\eta} v_{\rho}}$ \\
\hline \hline
\end{tabular}

$$
\begin{aligned}
V= & -\mu_{\chi}^{2}\left(\chi^{\dagger} \chi\right)-\mu_{\eta}^{2}\left(\eta^{\dagger} \eta\right)-\mu_{\rho}^{2}\left(\rho^{\dagger} \rho\right)+f\left(\eta_{i} \chi_{j} \rho_{k} \varepsilon^{i j k}+\text { H.c. }\right)+\lambda_{1}\left(\chi^{\dagger} \chi\right)\left(\chi^{\dagger} \chi\right)+\lambda_{2}\left(\rho^{\dagger} \rho\right)\left(\rho^{\dagger} \rho\right)+\lambda_{3}\left(\eta^{\dagger} \eta\right)\left(\eta^{\dagger} \eta\right) \\
& +\lambda_{4}\left(\chi^{\dagger} \chi\right)\left(\rho^{\dagger} \rho\right)+\lambda_{5}\left(\chi^{\dagger} \chi\right)\left(\eta^{\dagger} \eta\right)+\lambda_{6}\left(\rho^{\dagger} \rho\right)\left(\eta^{\dagger} \eta\right)+\lambda_{7}\left(\chi^{\dagger} \eta\right)\left(\eta^{\dagger} \chi\right)+\lambda_{8}\left(\chi^{\dagger} \rho\right)\left(\rho^{\dagger} \chi\right)+\lambda_{9}\left(\rho^{\dagger} \eta\right)\left(\eta^{\dagger} \rho\right),
\end{aligned}
$$

being $\chi, \rho$, and $\eta$, the $S U(3)_{L}$ scalar triplets.

The following relations arise from the global minimal conditions of the low energy scalar potential:

$$
\begin{aligned}
& \mu_{\chi}^{2}=-\frac{f v_{\eta} v_{\rho}}{\sqrt{2} v_{\chi}}+\frac{1}{2} \lambda_{5} v_{\eta}^{2}+\frac{1}{2} \lambda_{4} v_{\rho}^{2}+\lambda_{1} v_{\chi}^{2}, \\
& \mu_{\rho}^{2}=-\frac{f v_{\eta} v_{\chi}}{\sqrt{2} v_{\rho}}+\frac{1}{2} \lambda_{6} v_{\eta}^{2}+\lambda_{2} v_{\rho 2}^{2}+\frac{1}{2} \lambda_{4} v_{\chi}^{2}, \\
& \mu_{\eta}^{2}=-\frac{f v_{\rho} v_{\chi}}{\sqrt{2} v_{\eta}}+\lambda_{3} v_{\eta}^{2}+\frac{1}{2} \lambda_{6} v_{\rho}^{2}+\frac{1}{2} \lambda_{5} v_{\chi}^{2},
\end{aligned}
$$

From the scalar potential we find that the squared scalar mass matrices are

$$
\begin{aligned}
& M_{\zeta \zeta}^{2}=\left(\begin{array}{ccc}
\frac{f v_{\eta} v_{\rho}}{\sqrt{2} v_{\chi}} & \frac{f v_{\eta}}{\sqrt{2}} & \frac{f v_{\rho}}{\sqrt{2}} \\
\frac{f v_{\eta}}{\sqrt{2}} & \frac{f v_{\eta} v_{\chi}}{\sqrt{2} v_{\rho}} & \frac{f v_{\chi}}{\sqrt{2}} \\
\frac{f v_{\rho}}{\sqrt{2}} & \frac{f v_{\chi}}{\sqrt{2}} & \frac{f v_{0} v_{\chi}}{\sqrt{2} v_{\eta}}
\end{array}\right), \quad M_{\xi \xi}^{2}=\left(\begin{array}{ccc}
2 \lambda_{1} v_{\chi}^{2}+\frac{f v_{\eta} v_{\rho}}{\sqrt{2} v_{\chi}} & \lambda_{4} v_{\rho} v_{\chi}-\frac{f v_{\eta}}{\sqrt{2}} & \lambda_{5} v_{\eta} v_{\chi}-\frac{f v_{\rho}}{\sqrt{2}} \\
\lambda_{4} v_{\rho} v_{\chi}-\frac{f v_{\eta}}{\sqrt{2}} & 2 \lambda_{2} v_{\rho}^{2}+\frac{f v_{\eta} v_{\chi}}{\sqrt{2} v_{\rho}} & \lambda_{6} v_{\eta} v_{\rho}-\frac{f v_{\chi}}{\sqrt{2}} \\
\lambda_{5} v_{\eta} v_{\chi}-\frac{f v_{\rho}}{\sqrt{2}} & \lambda_{6} v_{\eta} v_{\rho}-\frac{f v_{\chi}}{\sqrt{2}} & 2 \lambda_{3} v_{\eta}^{2}+\frac{f v_{\rho} v_{\chi}}{\sqrt{2} v_{\eta}},
\end{array}\right), \\
& M_{\chi_{1}^{0} \eta_{3}^{0}}^{2}=M_{\bar{\chi}_{1}^{0} \bar{\eta}_{3}^{0}}^{2}=\left(\begin{array}{cc}
\lambda_{7} v_{\eta}^{2}+\frac{\sqrt{2} f v_{\rho} v_{\eta}}{v_{\chi}} & \sqrt{2} f v_{\rho}+\lambda_{7} v_{\eta} v_{\chi} \\
\sqrt{2} f v_{\rho}+\lambda_{7} v_{\eta} v_{\chi} & \lambda_{7} v_{\chi}^{2}+\frac{\sqrt{2} f v_{\rho} v_{\chi}}{v_{\eta}}
\end{array}\right), \quad M_{\eta_{2}^{ \pm} \rho_{1}^{ \pm}}^{2}=\left(\begin{array}{cc}
\lambda_{9} v_{\rho}^{2}+\frac{\sqrt{2} f v_{\chi} v_{\rho}}{v_{\eta}} & \lambda_{9} v_{\eta} v_{\rho}+\sqrt{2} f v_{\chi} \\
\lambda_{9} v_{\eta} v_{\rho}+\sqrt{2} f v_{\chi} & \lambda_{9} v_{\eta}^{2}+\frac{\sqrt{2} f v_{\chi} v_{\eta}}{v_{\rho}}
\end{array}\right) \text {, } \\
& M_{\chi_{2}^{ \pm} \rho_{3}^{ \pm}}^{2}=\left(\begin{array}{cc}
\lambda_{8} v_{\rho}^{2}+\frac{\sqrt{2} f v_{\eta} v_{\rho}}{v_{\chi}} & \sqrt{2} f v_{\eta}+\lambda_{8} v_{\rho} v_{\chi} \\
\sqrt{2} f v_{\eta}+\lambda_{8} v_{\rho} v_{\chi} & \lambda_{8} v_{\chi}^{2}+\frac{\sqrt{2} f v_{\eta} v_{\chi}}{v_{\rho}}
\end{array}\right) .
\end{aligned}
$$

The resulting physical scalars and their masses are given in Table IV. 


$$
\begin{aligned}
\tan (\alpha) & =\frac{v_{\eta}}{v_{\chi}} \\
\tan (\beta) & =\frac{v_{\rho}}{v_{\eta}} \\
\tan (\gamma) & =\frac{v_{\rho}}{v_{\chi}} \\
\tan (\delta) & =\frac{2\left(v_{\eta} \lambda_{6} v_{\rho}-\frac{f v_{\chi}}{\sqrt{2}}\right)}{\frac{f v_{\eta} v_{\chi}}{\sqrt{2} v_{\rho}}-\frac{f v_{\rho} v_{\chi}}{\sqrt{2} v_{\eta}}-2 v_{\eta}^{2} \lambda_{3}+2 \lambda_{2} v_{\rho}^{2}}
\end{aligned}
$$

The field composition of the low energy physical scalar spectrum of our model is given by one light neutral scalar $h^{0}$ identified with the SM-like $125.09 \mathrm{GeV}$ Higgs boson found at the LHC, five neutral heavy Higgs bosons $\left(H_{1}^{0}, H_{2}^{0}, H_{3}^{0}, \bar{H}_{3}^{0}, A^{0}\right)$ and four charged Higgs bosons $\left(H_{4}^{ \pm}, H_{5}^{ \pm}\right)$. It's worth mentioning that the neutral Goldstone bosons $G_{1}^{0}, G_{2}^{0}, G_{3}^{0}$ and $\bar{G}_{3}^{0}$ are related to the longitudinal components of the $Z, Z^{\prime}, K^{0}$ and $\bar{K}^{0}$ just like the charged Goldstone bosons $G_{1}^{ \pm}$and $G_{2}^{ \pm}$are related to the longitudinal components of the $W^{ \pm}$and $W^{\prime \pm}$ gauge bosons.

The $125 \mathrm{GeV}$ mass value for the SM-like Higgs boson can be reproduced for the following benchmark point:

$$
\begin{aligned}
& v_{\chi} \simeq 10 \mathrm{TeV}, \quad v_{\eta} \sim v_{\rho} \simeq 174 \mathrm{GeV}, \quad f \simeq 1000 \mathrm{GeV}, \\
& \lambda_{1} \simeq 0.016, \quad \lambda_{2} \sim \lambda_{3} \simeq 0.26, \quad \lambda_{4} \sim \lambda_{5} \sim \lambda_{7} \simeq 1, \\
& \lambda_{6} \simeq 10, \quad \lambda_{8} \simeq 0.01 .
\end{aligned}
$$

where in this scenario, the trilinear parameter $f$ has to be fixed at $1000 \mathrm{GeV}$ to get $M_{A^{0}} \simeq 5318.3 \mathrm{GeV}$ and $M_{H^{ \pm}} \simeq 5503.95 \mathrm{GeV}$.

\section{QUARK MASSES AND MIXINGS}

From the Yukawa interactions of the quark sector given by Eq. (2.5), we find that the SM quark mass matrices read:

$$
\begin{aligned}
M_{U} & =\frac{v}{\sqrt{2}}\left(\begin{array}{ccc}
c_{1} \lambda^{8} & 0 & a_{1} \lambda^{4} \\
0 & b_{2} \lambda^{4} & 0 \\
0 & 0 & a_{2}
\end{array}\right), \\
M_{D} & =\frac{v}{\sqrt{2}}\left(\begin{array}{ccc}
g_{1} \lambda^{7} & g_{4} \lambda^{6} & 0 \\
0 & g_{2} \lambda^{5} & 2 g_{2} \lambda^{5} \\
0 & 0 & g_{3} \lambda^{3}
\end{array}\right),
\end{aligned}
$$

\begin{tabular}{|c|c|c|c|}
\hline Observable & $\begin{array}{l}\text { Model } \\
\text { value with } \\
\text { Eq. (3.3) }\end{array}$ & $\begin{array}{c}\text { Model } \\
\text { value with } \\
\text { Eq. (3.4) }\end{array}$ & $\begin{array}{c}\text { Experimental } \\
\text { value }\end{array}$ \\
\hline$m_{u}(\mathrm{MeV})$ & 1.44999 & 1.44999 & $1.45_{-0.45}^{+0.56}$ \\
\hline$m_{c}(\mathrm{MeV})$ & 635 & 635 & $635 \pm 86$ \\
\hline$m_{t}(\mathrm{GeV})$ & 172.101 & 172.101 & $172.1 \pm 0.6 \pm 0.9$ \\
\hline$m_{d}(\mathrm{MeV})$ & 2.89988 & 2.90313 & $2.9_{-0.4}^{+0.5}$ \\
\hline$m_{s}(\mathrm{MeV})$ & 59.1145 & 60.021 & $57.7_{-15.8}^{+16.8}$ \\
\hline$m_{b}(\mathrm{GeV})$ & 2.79418 & 2.82003 & $2.82_{-0.04}^{+0.09}$ \\
\hline $\sin \theta_{12}$ & 0.225402 & 0.220611 & 0.225 \\
\hline $\sin \theta_{23}$ & 0.0412799 & 0.0415761 & 0.0412 \\
\hline $\sin \theta_{13}$ & 0.00386484 & 0.0038648 & 0.00351 \\
\hline$\delta_{q}$ & $68.021^{\circ}$ & $68.0198^{\circ}$ & $68^{\circ}$ \\
\hline
\end{tabular}

where $\lambda=0.225$ and $v=246 \mathrm{GeV}$. In order to get quark mixing angles and a $C P$ violating phase consistent with the experimental data, we assume that all dimensionless parameters given in Eq. (3.1) are real, except for $a_{1}$, taken to be complex.
TABLE V. Model and experimental values of the quark masses and CKM parameters.

The exotic quark masses are

$$
\begin{aligned}
& m_{t^{\prime}}=y^{\left(t^{\prime}\right)} \frac{v_{\chi}}{\sqrt{2}}, \quad m_{j_{1}}=y_{1}^{(j)} \frac{v_{\chi}}{\sqrt{2}}=\frac{y_{1}^{(j)}}{y^{\left(t^{\prime}\right)}} m_{t^{\prime}}, \\
& m_{j_{2}}=y_{2}^{(j)} \frac{v_{\chi}}{\sqrt{2}}=\frac{y_{2}^{(j)}}{y^{\left(t^{\prime}\right)}} m_{t^{\prime}} .
\end{aligned}
$$

The experimental values of the physical quark mass spectrum $[105,106]$, mixing angles and Jarlskog invariant [107] are consistent with their experimental data, as shown in Table V, starting from the following benchmark point:

$$
\begin{aligned}
& c_{1} \simeq 1.2525, \quad\left|a_{1}\right| \simeq 1.48406, \quad \arg \left(a_{1}\right) \simeq 68^{\circ}, \\
& a_{2} \simeq 0.989375, \quad b_{2} \simeq 1.41504 \text {, } \\
& g_{1} \simeq 0.579397, \quad g_{2} \simeq 0.57, \quad g_{3} \simeq 1.40209 \text {, } \\
& g_{4} \simeq 0.583 \text {. }
\end{aligned}
$$

The result given in Eq. (3.3) motivates to consider the simplified benchmark scenario:

$$
\begin{aligned}
& c_{1} \simeq 1.2525, \quad\left|a_{1}\right| \simeq 1.48406, \quad \arg \left(a_{1}\right) \simeq 68^{\circ}, \\
& a_{2} \simeq 0.989375, \\
& g_{1} \simeq g_{2} \simeq g_{4} \simeq 0.579397, \quad b_{2} \simeq g_{3} \simeq 1.41504 .
\end{aligned}
$$

Notice that a successful fit of the ten physical observables in the quark sector can be obtained in the above described scenarios where the first [Eq. (3.3)] and the second one [Eq. (3.4)] only have 9 and 6 effective free parameters, respectively. It is worth mentioning that in the general scenario of 9 effective free parameters, such parameters fit the CKM quark mixing matrix as well as 5 of the 6 quark masses, whereas the remaining quark mass is predicted. In the concerning to the scenario of 6 effective free parameters, the quark mixing angle $\theta_{13}$, the quark $C P$ violating phase $\delta$ and 2 quark masses are adjusted, whereas the 


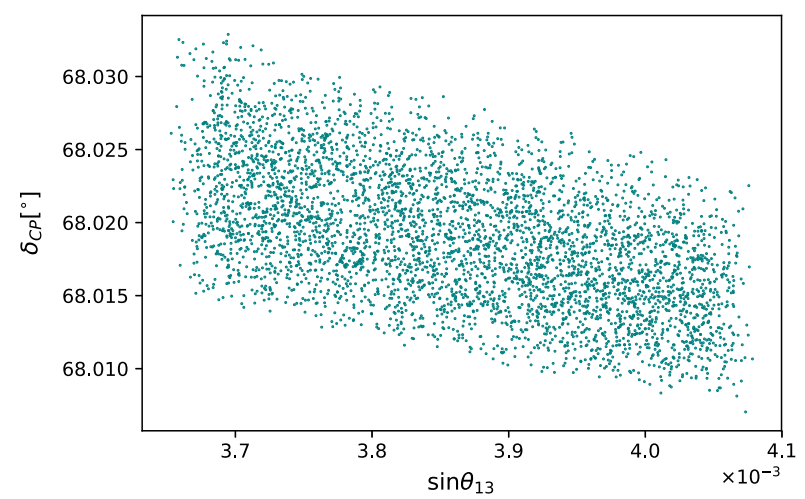

(a)

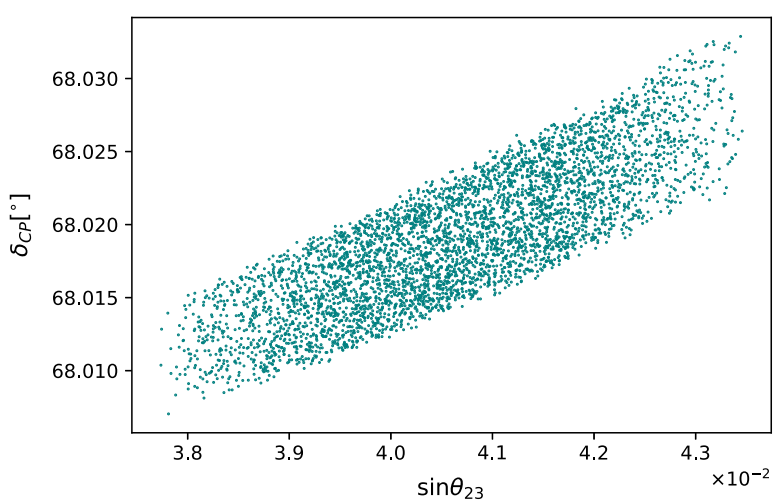

(b)

FIG. 1. Correlations between the quark $C P$-violating phase $\delta_{C P}$ and the quark mixing parameters $\sin \theta_{13}, \sin \theta_{23}$.

remaining quark mixing angles $\theta_{12}, \theta_{23}$ and 4 quark masses are predicted.

Thus, the symmetries of our model give rise to quark mass matrix textures that successfully explain the SM quark mass spectrum and mixing parameters, with quark sector effective free parameters of order unity.

In addition to the benchmark points of Eqs. (3.3) and (3.4), correlation plots in Fig. 1 have been obtained to analyze the behavior of some of the quark observables, such as the $C P$ violating phase $\delta_{C P}$ as a function of the quark mixing parameters $\sin \theta_{13}$ and $\sin \theta_{23}$.

These plots were generated by varying the quark sector parameters in Eq. (3.3) in a range of values that satisfies the $3 \sigma$ experimental allowed values in the quark sector and $0.224<\sin \theta_{12}<0.226$. The plots show that the $C P$ violating phase is predicted to be in range $68.007^{\circ} \lesssim \delta_{C P} \lesssim$ $68.032^{\circ}$ for the allowed parameter space. Figure 1(a) shows that $0.0036 \lesssim \sin \theta_{13} \lesssim 0.0040$ and as $\sin \theta_{13}$ grows up $\delta_{C P}$ goes down. On the other hand, Fig. 1(b) shows that $0.0377 \lesssim$ $\sin \theta_{23} \lesssim 0.0434$ and $\delta_{C P}$ is directly proportional to $\sin \theta_{23}$.

Finally to close this section we briefly comment about the LHC signatures of exotic $t^{\prime}, j_{1}$ and $j_{2}$ quarks in our model. Such exotic quarks will mainly decay into a top quark and either neutral or charged scalar and can be pair produced at the LHC via Drell-Yan and gluon fusion processes mediated by charged gauge bosons and gluons, respectively. A detailed study of the collider phenomenology of the model is beyond the scope of this paper and is left for future studies.

\section{LEPTON MASSES AND MIXINGS}

From Eq. (2.6), and using the product rules of the $S_{4}$ group given in Appendix A, we find that the charged lepton mass matrix is given by:

$$
M_{l}=\frac{v}{\sqrt{2}}\left(\begin{array}{ccc}
x_{1} \lambda^{9} & x_{4} \lambda^{5} & x_{5} \lambda^{3} \\
0 & x_{2} \lambda^{5} & x_{6} \lambda^{4} \\
0 & 0 & x_{3} \lambda^{3}
\end{array}\right)
$$

Regarding the neutrino sector, from Eq. (2.6), we find the following neutrino mass terms:

$$
-\mathcal{L}_{\text {mass }}^{(\nu)}=\frac{1}{2}\left(\begin{array}{lll}
\overline{\nu_{L}^{C}} & \overline{\nu_{R}} & \overline{N_{R}}
\end{array}\right) M_{\nu}\left(\begin{array}{c}
\nu_{L} \\
\nu_{R}^{C} \\
N_{R}^{C}
\end{array}\right)+\text { H.c. },
$$

where the neutrino mass matrix is given by:

$$
M_{\nu}=\left(\begin{array}{ccc}
0_{3 \times 3} & M_{1} & M_{2} \\
M_{1}^{T} & 0_{3 \times 3} & M_{3} \\
M_{2}^{T} & M_{3}^{T} & 0_{3 \times 3}
\end{array}\right),
$$

and the submatrices take the form:

$$
\begin{aligned}
& M_{1}=\frac{h_{\rho} v_{\rho} v_{\zeta}}{2 \Lambda}\left(\begin{array}{ccc}
0 & a & 0 \\
-a & 0 & b \\
0 & -b & 0
\end{array}\right), \\
& M_{2}=h_{\eta}^{(L)} \frac{v_{\eta} v_{\Sigma}}{\sqrt{6} \Lambda}\left(\begin{array}{ccc}
x & y & -y \\
-x & \omega^{2} y & -\omega y \\
x & \omega y & -\omega^{2} y
\end{array}\right), \\
& M_{3}=h_{\chi}^{(L)} \frac{v_{\chi} v_{\Sigma}}{\sqrt{6} \Lambda}\left(\begin{array}{ccc}
r & z & -z \\
-r & \omega^{2} z & -\omega z \\
r & \omega z & -\omega^{2} z
\end{array}\right), \quad \omega=e^{\frac{2 \pi i}{3}} .
\end{aligned}
$$

The light active neutrino masses arise from a linear seesaw mechanism and the physical neutrino mass matrices are

$$
\begin{gathered}
M_{\nu}^{(1)}=-\left[M_{2} M_{3}^{-1} M_{1}^{T}+M_{1}\left(M_{3}^{T}\right)^{-1} M_{2}^{T}\right], \\
M_{\nu}^{(2)}=-\frac{1}{2}\left(M_{3}+M_{3}^{T}\right)-\frac{1}{2}\left[M_{1}^{T} M_{1}\left(M_{3}^{T}\right)^{-1}+\left(M_{3}\right)^{-1} M_{1}^{T} M_{1}\right],
\end{gathered}
$$


$M_{\nu}^{(3)}=\frac{1}{2}\left(M_{3}+M_{3}^{T}\right)+\frac{1}{2}\left[M_{1}^{T} M_{1}\left(M_{3}^{T}\right)^{-1}+\left(M_{3}\right)^{-1} M_{1}^{T} M_{1}\right]$,

where $M_{\nu}^{(1)}$ corresponds to the active neutrino mass matrix whereas $M_{\nu}^{(2)}$ and $M_{\nu}^{(3)}$ are the sterile neutrino mass matrices. The physical neutrino spectrum is composed of 3 light active neutrinos and 6 nearly degenerate sterile exotic pseudoDirac neutrinos. Furthermore, from Eqs. (2.6) and (2.8) and considering $v_{\chi} \sim \mathcal{O}(10) \mathrm{TeV}, v_{\eta} \sim v_{\rho} \sim O(100) \mathrm{GeV}$ and the Yukawa couplings of order unity, we find that the light active neutrino mass scale $\sim 50 \mathrm{meV}$ is estimated as $m_{\nu} \sim \frac{v_{\eta} v_{p} v_{\zeta}}{v_{\chi} \Lambda} \sim \frac{v_{\eta} v_{\rho}}{\Lambda}$, which implies for the model cutoff the estimate $\Lambda \sim \mathcal{O}\left(10^{16}\right) \mathrm{GeV}$.

The sterile neutrinos can be produced in pairs at the LHC, via quark-antiquark annihilation mediated by a heavy $Z^{\prime}$ gauge boson. They can decay into SM particles giving rise to a SM charged lepton and a $W$ gauge boson in the final state. Thus, observing an excess of events with respect to the SM background in the opposite sign dileptons final states can be a signal in support of this model at the LHC. Studies of inverse seesaw neutrino signatures at colliders as well as the production of heavy neutrinos at the LHC are carried out in Refs. [108-122]. A detailed study of the implications of our model at colliders goes beyond the scope of this paper and is deferred for a future work.

The light active neutrino mass matrix is given by:

$$
M_{\nu}^{(1)}=\left(\begin{array}{ccc}
2 A & B e^{i \varphi}-2 A & A-B e^{i \varphi} \\
B e^{i \varphi}-2 A & 2\left(A-B e^{i \varphi}\right) & 2 B e^{i \varphi}-A \\
A-B e^{i \varphi} & 2 B e^{i \varphi}-A & -2 B e^{i \varphi}
\end{array}\right) .
$$

and the light active neutrino masses are

$$
\begin{aligned}
& m_{1}=0, \\
& m_{2}=\sqrt{2} \sqrt{5 A^{2}-2 \sqrt{6} \sqrt{A^{4}-3 A^{3} B \cos (\varphi)+A^{2} B^{2} \cos (2 \varphi)+3 A^{2} B^{2}-3 A B^{3} \cos (\varphi)+B^{4}}-7 A B \cos (\varphi)+5 B^{2},} \\
& m_{3}=\sqrt{2} \sqrt{5 A^{2}+2 \sqrt{6} \sqrt{A^{4}-3 A^{3} B \cos (\varphi)+A^{2} B^{2} \cos (2 \varphi)+3 A^{2} B^{2}-3 A B^{3} \cos (\varphi)+B^{4}}-7 A B \cos (\varphi)+5 B^{2},}
\end{aligned}
$$

which implies that the experimental values of the neutrino mass squared splittings can be very well reproduced for the following benchmark point:

$$
A=B=0.00949663 \mathrm{eV}, \quad \varphi=65.8796^{\circ} .
$$

The corresponding PMNS leptonic mixing matrix is defined as $U=R_{l}^{\dagger} R_{\nu}$, and from the standard parametrization of $U$, it follows that the lepton mixing parameters are given by:

$$
\begin{aligned}
& \sin ^{2}\left(\theta_{13}\right)=\left|U_{13}\right|^{2}, \quad \sin ^{2}\left(\theta_{12}\right)=\frac{\left|U_{12}\right|^{2}}{1-\left|U_{13}\right|^{2}}, \\
& \sin ^{2}\left(\theta_{23}\right)=\frac{\left|U_{23}\right|^{2}}{1-\left|U_{13}\right|^{2}} .
\end{aligned}
$$

It is worth mentioning that due to the complexity of the expression for the PMNS matrix, the analytic form cannot be shown.

Furthermore, the Jarlskog invariant $J_{C P}$ is determined from the relation:

$$
J_{C P}=\operatorname{Im}\left(U_{11}^{*} U_{23}^{*} U_{13} U_{21}\right),
$$

whereas the leptonic Dirac $C P$ violating phase $\delta_{C P}$ can be extracted from the equivalent definition of $J_{C P}$ [123] in the standard parametrization:
$J_{C P}=\frac{1}{8} \sin \left(2 \theta_{12}\right) \sin \left(2 \theta_{23}\right) \sin \left(2 \theta_{13}\right) \cos \left(\theta_{13}\right) \delta_{C P}$.

The charged lepton masses, leptonic mixing parameters and $C P$-phase can be very well reproduced for the scenario of normal neutrino mass ordering in terms of natural parameters of order one, as shown in Table VI, starting from the following benchmark point:

$x_{1}=-0.85677-2.19346 i, \quad x_{2}=-2.84582-1.22066 i$,

$x_{3}=-0.235108-0.00451549 i$,

$x_{4}=0.979847+2.04567 i, \quad x_{5}=0.220533+0.440278 i$,

$x_{6}=-2.69931-1.24421 i$.

As indicated by Table VI, our model is consistent with the experimental data on lepton masses and mixings. Notice that the ranges for the experimental values in Table VI were taken from [124] for the case of normal hierarchy. Note that we only consider the case of normal hierarchy since it is favored over more than $3 \sigma$ than the inverted neutrino mass ordering.

Figure 2 shows the correlations of the leptonic Dirac $C P$ violating phase $\delta_{C P}$ with the solar $\sin ^{2} \theta_{12}$ and with the reactor $\sin ^{2} \theta_{13}$ mixing parameters as well as the correlations between the leptonic mixing parameters. To obtain these figures, the lepton sector parameters were randomly 
TABLE VI. Model and experimental values for the physical observables of the neutrino sector: neutrino mass squared splittings, leptonic mixing angles and the leptonic $C P$ phase for the scenario of normal ordering.

\begin{tabular}{lcccc}
\hline \hline & & \multicolumn{2}{c}{ Experimental value } \\
\cline { 2 - 5 } Observable & Model Value & $1 \sigma$ range & $2 \sigma$ range & 0.487 \\
\hline$m_{e}[\mathrm{MeV}]$ & 0.487 & 0.487 & $102.8 \pm 0.0006$ & $102.8 \pm 0.0009$ \\
$m_{\mu}[\mathrm{MeV}]$ & 102.8 & $102.8 \pm 0.0003$ & $1.75 \pm 0.0006$ & $1.75 \pm 0.0009$ \\
$m_{\tau}[\mathrm{GeV}]$ & 1.75 & $1.75 \pm 0.0003$ & $7.20-7.94$ & $7.05-8.14$ \\
$\Delta m_{21}^{2}\left[10^{-5} e V^{2}\right]$ & 7.55 & $7.55_{-0.16}^{+0.20}$ & $2.44-2.57$ & $2.41-2.60$ \\
$\Delta m_{31}^{2}\left[10^{-3} e V^{2}\right]$ & 2.50 & $2.50 \pm 0.03$ & $2.89-3.59$ & $2.73-3.79$ \\
$\sin ^{2}\left(\theta_{12}\right) / 10^{-1}$ & 3.20 & $3.20_{-0.16}^{+0.20}$ & $4.67-5.83$ & $4.45-5.99$ \\
$\sin ^{2}\left(\theta_{23}\right) / 10^{-1}$ & 5.47 & $5.47_{-0.30}^{+0.20}$ & $2.03-2.34$ & $1.96-2.41$ \\
$\sin ^{2}\left(\theta_{13}\right) / 10^{-2}$ & 2.160 & $2.160_{-0.069}^{+0.083}$ & $212^{\circ}-315^{\circ}$ \\
$\delta_{C P}^{+38^{\circ}}$ & $248.78^{\circ}$ & $157^{\circ}-349^{\circ}$ \\
\hline \hline
\end{tabular}

generated in a range of values where the neutrino mass squared splittings, leptonic mixing parameters and leptonic Dirac $C P$ violating phase are inside the $3 \sigma$ experimentally allowed range. We found a leptonic Dirac $C P$ violating

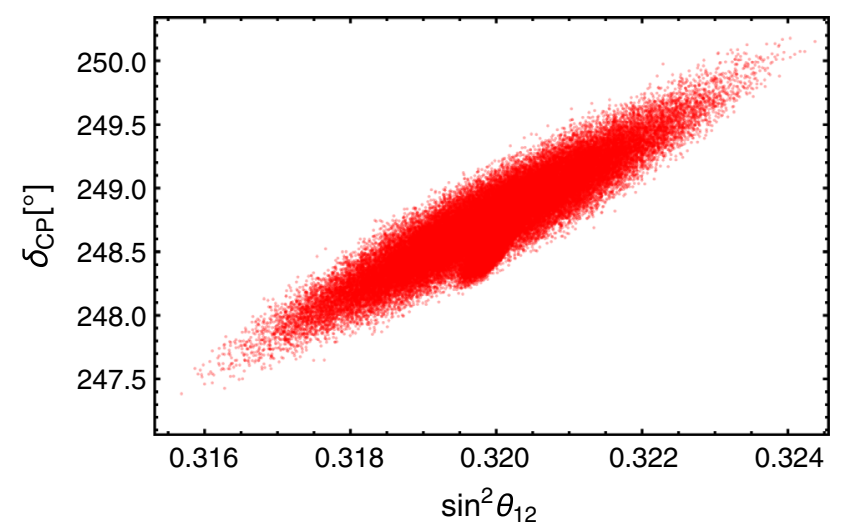

(a)

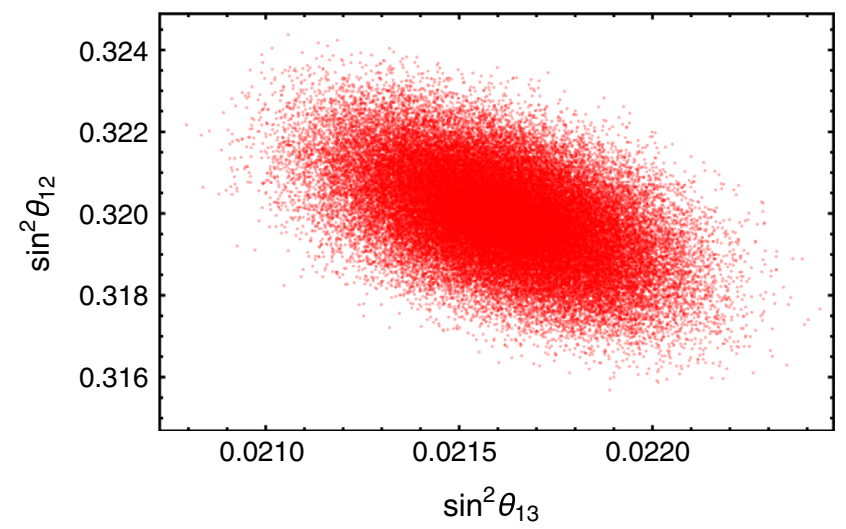

(c) phase in the range $247.5^{\circ} \lesssim \delta_{C P} \lesssim 250.2^{\circ}$, whereas the leptonic mixing parameters are obtained to be in the ranges $0.316 \lesssim \sin ^{2} \theta_{12} \lesssim 0.324,0.5462 \lesssim \sin ^{2} \theta_{23} \lesssim 0.5476$ and $0.0208 \lesssim \sin ^{2} \theta_{13} \lesssim 0.0224$.

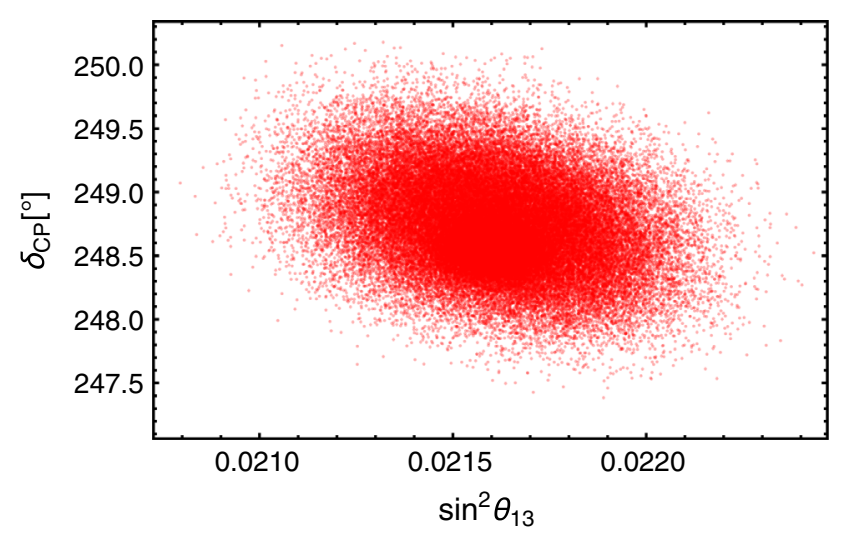

(b)

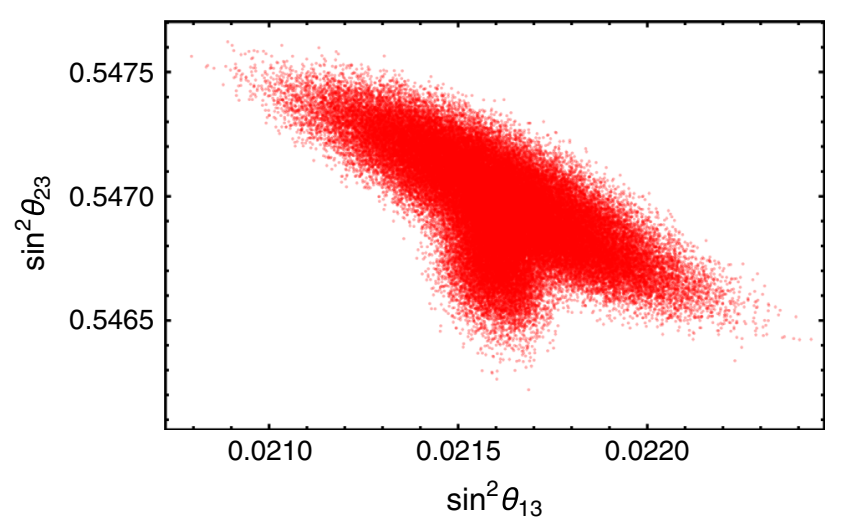

(d)

FIG. 2. Correlations between the different lepton sector observables. 


\section{HIGGS DIPHOTON DECAY RATE CONSTRAINTS}

The decay rate expression for the $h \rightarrow \gamma \gamma$ process is given by [125-132]:

$$
\begin{aligned}
\Gamma(h \rightarrow \gamma \gamma)= & \frac{\alpha_{e m}^{2} m_{h}^{3}}{256 \pi^{3} v^{2}} \mid \sum_{f} a_{h f f} N_{C} Q_{f}^{2} F_{1 / 2}\left(\rho_{f}\right) \\
& +a_{h W W} F_{1}\left(\rho_{W}\right)+a_{h W^{\prime} W^{\prime}} F_{1}\left(\rho_{W^{\prime}}\right) \\
& +\left.\frac{\lambda_{h H^{ \pm} H^{\mp}} v}{2 m_{H^{ \pm}}^{2}} F_{0}\left(\rho_{H^{ \pm}}\right)\right|^{2},
\end{aligned}
$$

where

$$
\begin{gathered}
a_{h W W}=\sin (\beta-\delta), \\
a_{h W^{\prime} W^{\prime}}=\cos \delta \sin \gamma, \\
a_{h t t}=-\frac{\sin \delta}{\sin \beta}, \\
\lambda_{h H^{ \pm} H^{ \pm}}=2\left(-\lambda_{5} \sin (\delta) \sin ^{2}(\gamma) \nu_{\eta}\right. \\
+\lambda_{6}(-\sin (\delta)) \cos ^{2}(\gamma) \nu_{\eta}+\cos (\delta)\left(\nu_{\rho}\right. \\
+\left(\left(\lambda_{4}+\lambda_{8}\right) \sin ^{2}(\gamma)+2 \lambda_{2} \cos ^{2}(\gamma)\right) \\
\left.\left.+\lambda_{8} \sin (\gamma) \cos (\gamma) \nu_{\chi}\right)\right) .
\end{gathered}
$$

Here $\rho_{i}$ are the mass ratios $\rho_{i}=\frac{m_{h}^{2}}{4 M_{i}^{2}}$ with $M_{i}=m_{f}$, $M_{W}, M_{W^{\prime}} ; \alpha_{e m}$ is the fine structure constant; $N_{C}$ is the color factor $\left(N_{C}=1\right.$ for leptons and $N_{C}=3$ for quarks); and $Q_{f}$ is the electric charge of the fermion in the loop. From the fermion-loop contributions we only consider the dominant top quark term.

Furthermore, $F_{1 / 2}(z)$ and $F_{1}(z)$ are the dimensionless loop factors for spin-1/2 and spin-1 particles running in the internal lines of the loops. These loop factors take the form:

$$
\begin{gathered}
F_{1 / 2}(z)=2(z+(z-1) f(z)) z^{-2}, \\
F_{1}(z)=-2\left(2 z^{2}+3 z+3(2 z-1) f(z)\right) z^{-2}, \\
F_{0}(z)=-(z-f(z)) z^{-2},
\end{gathered}
$$

with

$$
f(z)= \begin{cases}\arcsin ^{2} \sqrt{2} & \text { for } \quad z \leq 1 \\ -\frac{1}{4}\left(\ln \left(\frac{1+\sqrt{1-z^{-1}}}{1-\sqrt{1-z^{-1}}-i \pi}\right)^{2}\right) & \text { for } z>1\end{cases}
$$

In order to get the constraints on the model parameter space arising from the decay of the $126 \mathrm{GeV}$ Higgs into a photon pair, the observable $R_{\gamma \gamma}$ is introduced:
TABLE VII. Parameters with $\nu_{\eta}=173.948 \mathrm{GeV}, \quad \nu_{\rho}=$ $173.948 \mathrm{GeV}$ and $\nu_{\chi}=10 \mathrm{TeV}$.

\begin{tabular}{lc}
\hline \hline Parameters & Model value \\
\hline$M_{h^{0}}$ & $125.09 \mathrm{GeV}$ \\
$M_{H^{0}}$ & $5319.77 \mathrm{GeV}$ \\
$M_{A^{0}}$ & $5318.3 \mathrm{GeV}$ \\
$M_{H^{ \pm}}$ & $5503.95 \mathrm{GeV}$ \\
$a_{h W^{-} W^{+}}$ & 1.0 \\
$a_{h W^{\prime} W^{\prime}}$ & 0.0122981 \\
$a_{h t t}$ & 1.0 \\
$\lambda_{h H^{ \pm} H^{ \pm}}$ & $2525.45 \mathrm{GeV}$ \\
\hline \hline
\end{tabular}

$R_{\gamma \gamma}=\frac{\sigma(p p \rightarrow h) \Gamma(h \rightarrow \gamma \gamma)}{\sigma(p p \rightarrow h)_{\mathrm{SM}} \Gamma(h \rightarrow \gamma \gamma)_{\mathrm{SM}}} \simeq a_{h t t}^{2} \frac{\Gamma(h \rightarrow \gamma \gamma)}{\Gamma(h \rightarrow \gamma \gamma)_{\mathrm{SM}}}$.

That observable, which is called the Higgs diphoton signal strength, normalizes the $\gamma \gamma$ signal predicted by our model in relation to the one given by the SM. We have used the same normalization as in Refs. [77,132-138]. The ratio $R_{\gamma \gamma}$ has been measured by CMS and ATLAS collaborations with the best fit signals $[139,140]$ :

$R_{\gamma \gamma}^{\mathrm{CMS}}=1.14_{-0.23}^{+0.26} \quad$ and $\quad R_{\gamma \gamma}^{\mathrm{ATLAS}}=1.17 \pm 0.27$.

The best fit result for the ratio $R_{\gamma \gamma}$ is

$$
R_{\gamma \gamma}=1.0267
$$

This value was obtained using the best fit results shown in Table VII and is consistent with the current Higgs diphoton decay rate constraints. Correlations plots have been obtained to observe the behavior of the $R_{\gamma \gamma}$ parameter as function of the scalar masses and $W^{\prime}$ gauge boson mass. They are shown in Fig. 3.

These plots were generated using random points in a space in the neighborhood of the best fit values for $f, v_{\chi}$, and $\lambda_{8}$. Figure 3(a) shows that the parameter $R_{\gamma \gamma}$ is strongly restricted by the $C P$-odd Higgs mass $M_{A^{0}}$, since the range of allowed values for $R_{\gamma \gamma}$ decreases when the $C P$ odd scalar mass $M_{A^{0}}$ is increased. The Higgs diphoton signal strength $R_{\gamma \gamma}$ features a similar behavior with the charged scalar mass $M_{H^{ \pm}}$, as indicated by Fig. 3(b). Notice that despite the $C P$ odd neutral scalar $A^{0}$ does not contribute to the Higgs diphoton decay rate, the Higgs diphoton signal strength indirectly depends on $M_{A^{0}}$ since the parameters $\delta, \gamma$, and $\lambda_{h H^{ \pm} H^{ \pm}}$(that enter in the Higgs diphoton decay rate) as well as the $C P$-odd Higgs mass $M_{A^{0}}$ are functions of $v_{\chi}$. In addition, we have found that the Higgs diphoton signal strength decreases when the $W^{\prime}$ mass is increased, approaching to 1 when $M_{W^{\prime}} \gtrsim 10 \mathrm{TeV}$, as indicated by Fig. 3(c). Furthermore, Figs. 3(a), 3(b), and 3(c) show that our model favors values for the Higgs diphoton decay rate 


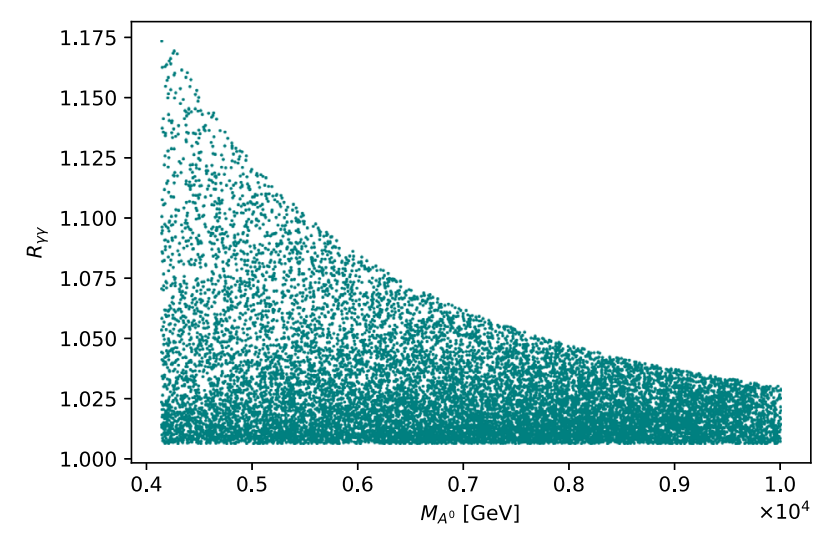

(a)

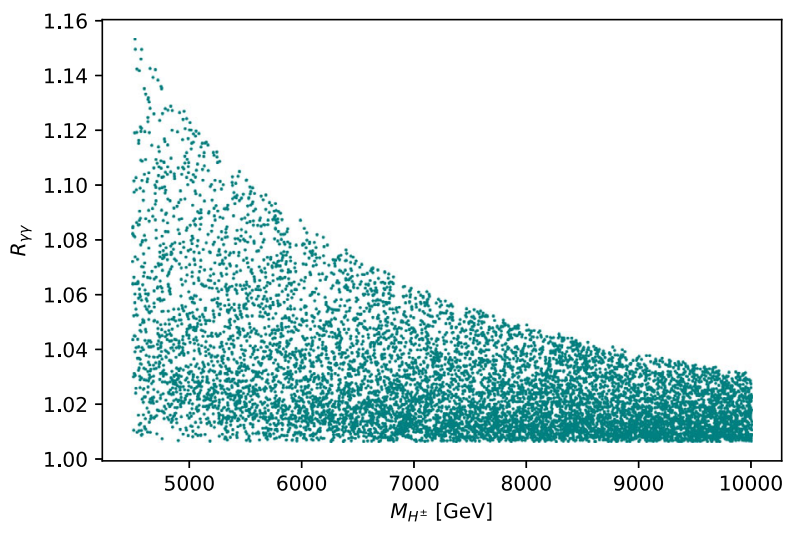

(b)

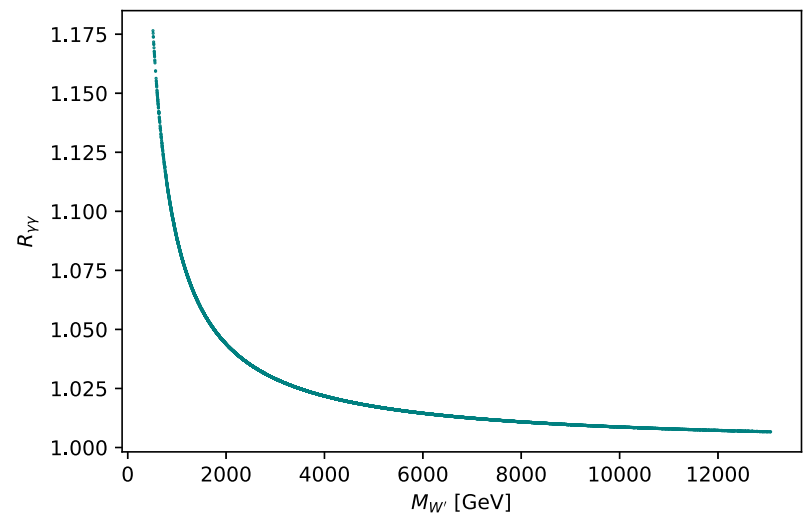

(c)

FIG. 3. Correlations of the $R_{\gamma \gamma}$ parameter with the masses of the $C P$-odd scalar, charged scalars, and $W^{\prime}$ gauge boson.

larger than the SM expectation. In addition, Fig. 4 shows that the Higgs diphoton decay rate constraints are fulfilled when $M_{H^{ \pm}} \gtrsim M_{A^{0}}$. Finally, our obtained results for the Higgs diphoton signal strength indicate that the Higgs diphoton decay is a smoking gun signature of our model, whose more precise measurement will be crucial to assess its viability.

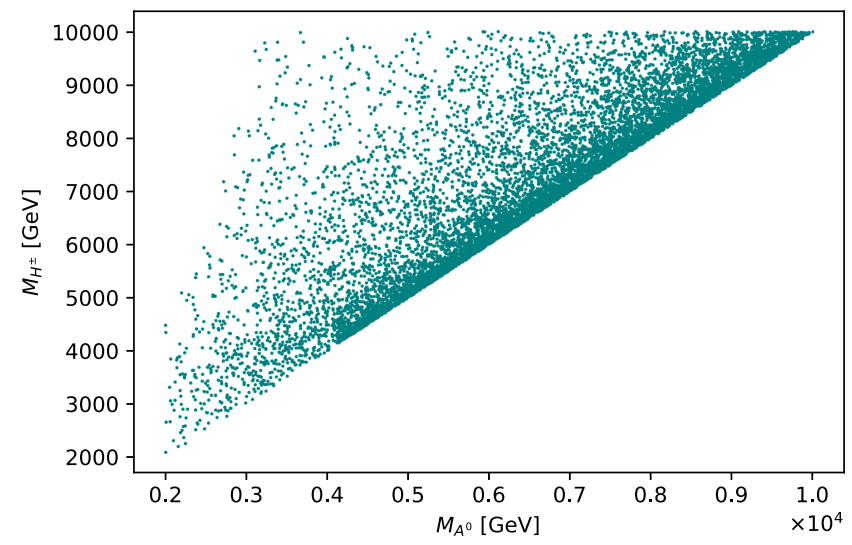

FIG. 4. Correlation plot of the $C P$-odd Higgs mass and the charged Higgs mass.

\section{HEAVY SCALAR PRODUCTION AT PROTON-PROTON COLLIDER}

In this section we discuss the singly heavy scalar $H_{1}$ production at proton-proton collider. It is worth mentioning that the production mechanism at the LHC of the heavy scalar $H_{1}$ is via gluon fusion, which is a one loop process mediated by the heavy exotic $t^{\prime}, j_{1}$, and $j_{2}$ quarks. Consequently, the total $H_{1}$ production cross section in proton collisions with center of mass energy $\sqrt{S}$ is given by:

$$
\begin{aligned}
\sigma_{p p \rightarrow g g \rightarrow H_{1}}(S) & \frac{\alpha_{S}^{2} m_{H_{1}}^{2}}{64 \pi v_{\chi}^{2} S}\left[I\left(\frac{m_{H_{1}}^{2}}{m_{t^{\prime}}^{2}}\right)+I\left(\frac{m_{H_{1}}^{2}}{m_{j_{1}}^{2}}\right)+I\left(\frac{m_{H_{1}}^{2}}{m_{j_{2}}^{2}}\right)\right] \\
& \times \int_{\ln \sqrt{\frac{m_{H_{1}}^{2}}{S}}}^{-\ln \sqrt{\frac{m_{H_{1}}^{2}}{S}}} f_{p / g}\left(\sqrt{\frac{m_{H_{1}}^{2}}{S} e^{y}, \mu^{2}}\right) \\
& \times f_{p / g}\left(\sqrt{\frac{m_{H_{1}}^{2}}{S}} e^{-y}, \mu^{2}\right) d y,
\end{aligned}
$$




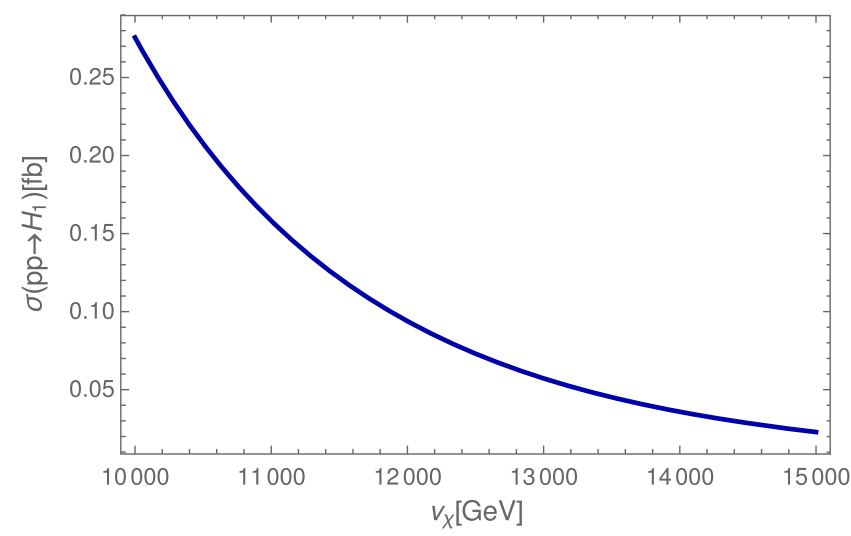

FIG. 5. Total cross section for the $H_{1}$ production via gluon fusion mechanism at the LHC for $\sqrt{S}=13 \mathrm{TeV}$ and as a function of the $S U(3)_{L} \times U(1)_{X}$ symmetry breaking scale $v_{\chi}$ for the scenario described in Eq. (2.20).

where $f_{p / g}\left(x_{1}, \mu^{2}\right)$ and $f_{p / g}\left(x_{2}, \mu^{2}\right)$ are the distributions of gluons in the proton which carry momentum fractions $x_{1}$ and $x_{2}$ of the proton, respectively. Furthermore $\mu=m_{H_{1}}$ is the factorization scale and $I(z)$ is given by:

$$
I(z)=\int_{0}^{1} d x \int_{0}^{1-x} d y \frac{1-4 x y}{1-z x y} .
$$

Figure 5 displays the $H_{1}$ total production cross section at the LHC via gluon fusion mechanism for $\sqrt{S}=13 \mathrm{TeV}$, as a function of the $S U(3)_{L} \times U(1)_{X}$ symmetry breaking scale $v_{\chi}$, which is taken to range from $10 \mathrm{TeV}$ up to $15 \mathrm{TeV}$, which corresponds to a heavy scalar mass $m_{H_{1}}$ varying between $1.3 \mathrm{TeV}$ and $1.9 \mathrm{TeV}$. In addition, the exotic quark Yukawa couplings have been taken equal to unity and the scenario described by Eq. (2.20) has been considered in our numerical analysis. Notice that the $S U(3)_{L} \times U(1)_{X}$

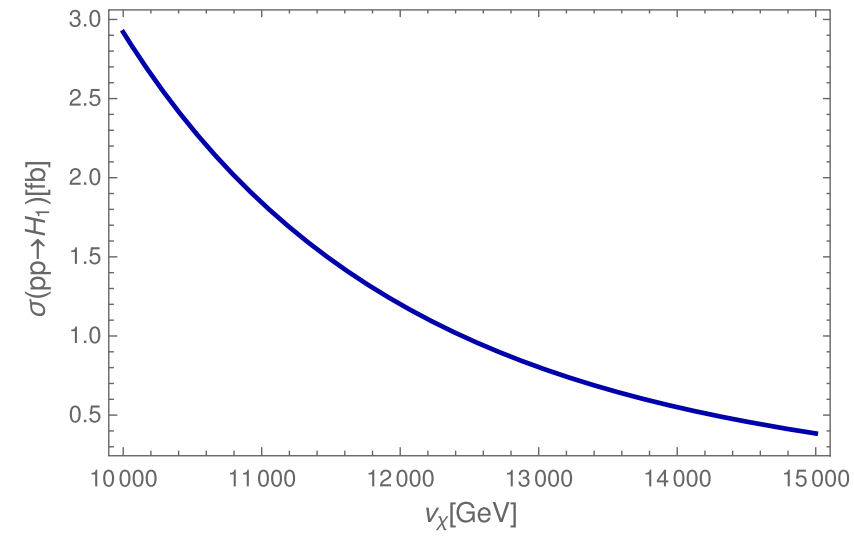

FIG. 6. Total cross section for the $H_{1}$ production via gluon fusion mechanism at the proposed energy upgrade of the LHC with $\sqrt{S}=28 \mathrm{TeV}$ as a function of the $S U(3)_{L} \times U(1)_{X}$ symmetry breaking scale $v_{\chi}$ for the scenario described in Eq. (2.20).

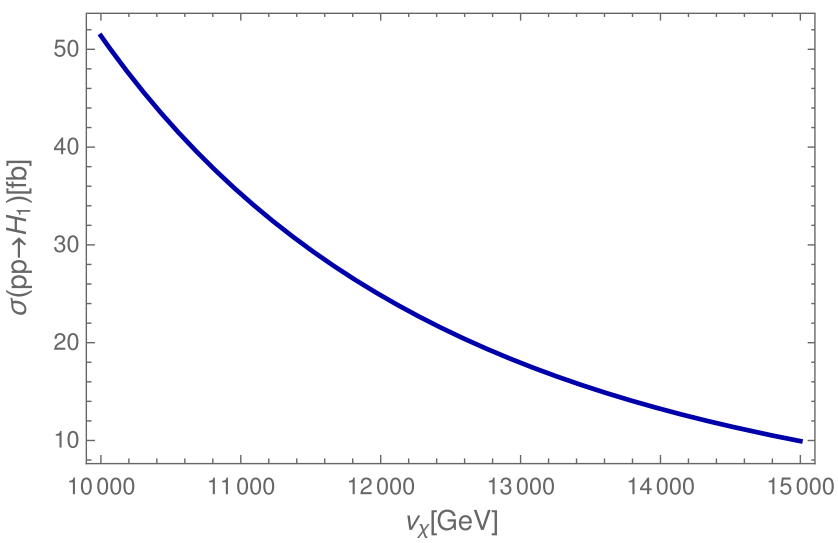

FIG. 7. Total cross section for the $H_{1}$ production via gluon fusion mechanism at a $\sqrt{S}=100 \mathrm{TeV}$ proton-proton collider as a function of the $S U(3)_{L} \times U(1)_{X}$ symmetry breaking scale $v_{\chi}$ for the scenario described in Eq. (2.20).

symmetry breaking scale has been taken larger than $10 \mathrm{TeV}$, which corresponds to a $Z^{\prime}$ gauge boson heavier than $4 \mathrm{TeV}$, in order to comply with the experimental data on $K, D$, and $B$ meson mixings [96]. For such region of $H_{1}$ masses, we find that the total production cross section is found to be $0.28-0.02 \mathrm{fb}$. However, at the proposed energy upgrade of the LHC with $\sqrt{S}=28 \mathrm{TeV}$, the $H_{1}$ production cross section is enlarged, reaching values of $2.9-0.4 \mathrm{fb}$ in the same mass region as indicated by Fig. 6 . Such small values for the $H_{1}$ production cross section at a proton-proton collider with $\sqrt{S}=13 \mathrm{TeV}$ and $\sqrt{S}=$ $28 \mathrm{TeV}$ are small to give rise to a signal for the relevant region of parameter space. However at a $\sqrt{S}=100 \mathrm{TeV}$ proton-proton collider, there is a significant enhancement of the $H_{1}$ production cross section, which takes values of $51-10 \mathrm{fb}$ for $1.3 \mathrm{TeV} \lesssim m_{H_{1}} \lesssim 1.9 \mathrm{TeV}$, as shown in Fig. 7. Finally, it is worth mentioning that one can safely assume that the heavy $H_{1}$ scalar after being produced will mainly decay into a pair of SM Higgs bosons, since it is the lightest non-SM scalar, as follows from Eq. (2.20) and Table IV. Consequently, an enhancement of the SM Higgs pair production with respect to the SM expectation, will be a smoking gun signature of this model, whose observation will be crucial to assess its viability.

\section{CONCLUSIONS}

We have constructed a multiscalar singlet extension of the 3-3-1 model with three right handed Majorana neutrinos, consistent with the observed SM fermion mass and mixing pattern. The model incorporates the $S_{4}$ family symmetry, which is combined with other auxiliary symmetries, thus allowing a viable description of the current SM fermion mass and mixing pattern, which is generated by the spontaneous breaking of the discrete group. The small masses of the light active neutrinos are produced by a 
linear seesaw mechanism mediated by three Majorana neutrinos. The model provides a successful fit of the physical observables of both quark and lepton sectors. Our model is predictive in the SM quark sector, since it only has 9 effective parameters that allow a successful fit of its 10 observables, i.e., the 6 SM quark masses, the 3 quark mixing parameters and the $C P$ violating phase. In addition, we have found that the SM quark sector of our model has a particular scenario, which is inspired by naturalness arguments and has only 6 effective parameters that allows to successfully reproduce the experimental values of the ten SM quark sector observables. Furthermore, we have also shown that the proposed model successfully accommodates the current Higgs diphoton decay rate constraints provided that the charged Higgs bosons are a bit heavier than the $\mathrm{CP}$ odd neutral Higgs boson $A^{0}$. In addition, we have found that it favors a Higgs diphoton decay rate larger than the SM expectation. Finally, we have also discussed the single production

of the heavy scalar $H_{1}$ associated with the spontaneous breaking of the $S U(3)_{C} \times U(1)_{X}$ symmetry, at a protonproton collider, via gluon fusion mechanism. We have considered the cases where the center of mass energy takes the values of $\sqrt{S}=13 \mathrm{TeV}, \sqrt{S}=28 \mathrm{TeV}$, and $\sqrt{S}=100 \mathrm{TeV}$. For the first two cases corresponding to the current LHC center of mass energy and the proposed energy upgrade of the LHC, respectively, we have found that the $H_{1}$ production cross sections are small to give rise to a signal for the relevant region of parameter space. However, in a future $\sqrt{S}=100 \mathrm{TeV}$ proton-proton collider, the $H_{1}$ production cross section is significantly enhanced reaching values between $51 \mathrm{fb}$ and $10 \mathrm{fb}$, for the mass range $1.3 \mathrm{TeV} \lesssim m_{H_{1}} \lesssim 1.9 \mathrm{TeV}$.

\section{ACKNOWLEDGMENTS}

This research has received funding from Fondecyt (Chile), Grants No. 1170803, CONICYT PIA/Basal FB0821 and the Programa de Incentivos a la Iniciación Científica (PIIC) from USM. A. E. C.H is very grateful to Professor Hoang Ngoc Long for hospitality at the Institute of Physics, Vietnam Academy of Science and Technology, where this work was finished.

\section{APPENDIX A: THE $S_{4}$ DISCRETE GROUP}

The $S_{4}$ is the smallest non-Abelian group having doublet, triplet, and singlet irreducible representations. $S_{4}$ is the group of permutations of four objects, which includes five irreducible representations, i.e., $\mathbf{1}, \mathbf{1}^{\prime}, \mathbf{2}, \mathbf{3}, \mathbf{3}^{\prime}$ fulfilling the following tensor product rules [141]:

$$
3 \otimes 3=1 \oplus 2 \oplus 3 \oplus 3^{\prime}, \quad 3^{\prime} \otimes 3^{\prime}=1 \oplus 2 \oplus 3 \oplus 3^{\prime}, \quad 3 \otimes 3^{\prime}=1^{\prime} \oplus 2 \oplus 3 \oplus 3^{\prime},
$$

$$
\begin{array}{cl}
2 \otimes 2=1 \oplus 1^{\prime} \oplus 2, & 2 \otimes 3=3 \oplus 3^{\prime}, \quad 2 \otimes 3^{\prime}=3^{\prime} \oplus 3, \\
3 \otimes 1^{\prime}=3^{\prime}, & 3^{\prime} \otimes 1^{\prime}=3, \quad 2 \otimes 1^{\prime}=2 .
\end{array}
$$

Explicitly, the basis used in this paper corresponds to Ref. [141] and results in

$$
\begin{gathered}
(\mathbf{A})_{\mathbf{3}} \times(\mathbf{B})_{\mathbf{3}}=(\mathbf{A} \cdot \mathbf{B})_{\mathbf{1}}+\left(\begin{array}{c}
\mathbf{A} \cdot \Sigma \cdot \mathbf{B} \\
\mathbf{A} \cdot \Sigma^{*} \cdot \mathbf{B}
\end{array}\right)_{\mathbf{2}}+\left(\begin{array}{l}
\left\{A_{y} B_{z}\right\} \\
\left\{A_{z} B_{x}\right\} \\
\left\{A_{x} B_{y}\right\}
\end{array}\right)_{\mathbf{3}}+\left(\begin{array}{l}
{\left[A_{y} B_{z}\right]} \\
{\left[A_{z} B_{x}\right]} \\
{\left[A_{x} B_{y}\right]}
\end{array}\right)_{\mathbf{3}^{\prime}} \\
(\mathbf{A})_{\mathbf{3}^{\prime}} \times(\mathbf{B})_{\mathbf{3}^{\prime}}=(\mathbf{A} \cdot \mathbf{B})_{\mathbf{1}}+\left(\begin{array}{c}
\mathbf{A} \cdot \Sigma \cdot \mathbf{B} \\
\mathbf{A} \cdot \Sigma^{*} \cdot \mathbf{B}
\end{array}\right)_{\mathbf{2}}+\left(\begin{array}{l}
\left\{A_{y} B_{z}\right\} \\
\left\{A_{z} B_{x}\right\} \\
\left\{A_{x} B_{y}\right\}
\end{array}\right)_{\mathbf{3}}+\left(\begin{array}{l}
{\left[A_{y} B_{z}\right]} \\
{\left[A_{z} B_{x}\right]} \\
{\left[A_{x} B_{y}\right]}
\end{array}\right)_{\mathbf{3}^{\prime}} \\
(\mathbf{A})_{\mathbf{3}} \times(\mathbf{B})_{\mathbf{3}^{\prime}}=(\mathbf{A} \cdot \mathbf{B})_{\mathbf{1}^{\prime}}+\left(\begin{array}{c}
\mathbf{A} \cdot \Sigma \cdot \mathbf{B} \\
-\mathbf{A} \cdot \Sigma^{*} \cdot \mathbf{B}
\end{array}\right)_{\mathbf{2}}+\left(\begin{array}{l}
\left\{A_{y} B_{z}\right\} \\
\left\{A_{z} B_{x}\right\} \\
\left\{A_{x} B_{y}\right\}
\end{array}\right)_{\mathbf{3}^{\prime}}+\left(\begin{array}{l}
{\left[A_{y} B_{z}\right]} \\
{\left[A_{z} B_{x}\right]} \\
{\left[A_{x} B_{y}\right]}
\end{array}\right)_{\mathbf{3}} \\
\left(\begin{array}{l}
\mathbf{A})_{\mathbf{2}} \times(\mathbf{B})_{\mathbf{2}}=\left\{A_{x} B_{y}\right\}_{\mathbf{1}}+\left[A_{x} B_{y}\right]_{\mathbf{1}^{\prime}}+\left(\begin{array}{c}
A_{y} B_{y} \\
A_{x} B_{x}
\end{array}\right)_{\mathbf{2}} \\
\left.\left(\begin{array}{l}
A_{x} \\
A_{y}
\end{array}\right)_{\mathbf{2}} \times\left(\begin{array}{l}
B_{x} \\
B_{y} \\
B_{z}
\end{array}\right)_{\mathbf{3}}=\left(\begin{array}{c}
\left(A_{x}+A_{y}\right) B_{x} \\
\left(\omega^{2} A_{x}+\omega A_{y}\right) B_{y} \\
\left(\omega A_{x}+\omega^{2} A_{y}\right) B_{z}
\end{array}\right)_{\mathbf{3}} \begin{array}{c}
\left(A_{x}-A_{y}\right) B_{x} \\
\left(\omega^{2} A_{x}-\omega A_{y}\right) B_{y} \\
\left(\omega A_{x}-\omega^{2} A_{y}\right) B_{z}
\end{array}\right)_{\mathbf{3}^{\prime}}
\end{array}\right.
\end{gathered}
$$




$$
\begin{aligned}
\left(\begin{array}{l}
A_{x} \\
A_{y}
\end{array}\right)_{2} \times\left(\begin{array}{c}
B_{x} \\
B_{y} \\
B_{z}
\end{array}\right)_{\mathbf{3}^{\prime}}= & \left(\begin{array}{c}
\left(A_{x}+A_{y}\right) B_{x} \\
\left(\omega^{2} A_{x}+\omega A_{y}\right) B_{y} \\
\left(\omega A_{x}+\omega^{2} A_{y}\right) B_{z}
\end{array}\right)_{\mathbf{3}^{\prime}} \\
& +\left(\begin{array}{c}
\left(A_{x}-A_{y}\right) B_{x} \\
\left(\omega^{2} A_{x}-\omega A_{y}\right) B_{y} \\
\left(\omega A_{x}-\omega^{2} A_{y}\right) B_{z}
\end{array}\right)_{3},
\end{aligned}
$$

with

$$
\begin{aligned}
\mathbf{A} \cdot \mathbf{B} & =A_{x} B_{x}+A_{y} B_{y}+A_{z} B_{z}, \\
\left\{A_{x} B_{y}\right\} & =A_{x} B_{y}+A_{y} B_{x}, \\
{\left[A_{x} B_{y}\right] } & =A_{x} B_{y}-A_{y} B_{x}, \\
\mathbf{A} \cdot \Sigma \cdot \mathbf{B} & =A_{x} B_{x}+\omega A_{y} B_{y}+\omega^{2} A_{z} B_{z}, \\
\mathbf{A} \cdot \Sigma^{*} \cdot \mathbf{B} & =A_{x} B_{x}+\omega^{2} A_{y} B_{y}+\omega A_{z} B_{z},
\end{aligned}
$$

where $\omega=e^{2 \pi i / 3}$ is a complex square root of unity.

\section{APPENDIX B: THE SCALAR POTENTIAL FOR A $S_{4}$ DOUBLET}

The scalar potential for a $S_{4}$ doublet $\Delta$ is given by:

$$
\begin{aligned}
V= & -\mu_{\Delta}^{2}\left(\Delta \Delta^{*}\right)_{\mathbf{1}}+\kappa_{1}\left(\Delta \Delta^{*}\right)_{\mathbf{1}}\left(\Delta \Delta^{*}\right)_{\mathbf{1}}+\kappa_{2}\left(\Delta \Delta^{*}\right)_{\mathbf{1}^{\prime}}\left(\Delta \Delta^{*}\right)_{\mathbf{1}^{\prime}} \\
& +\kappa_{3}\left(\Delta \Delta^{*}\right)_{\mathbf{2}}\left(\Delta \Delta^{*}\right)_{\mathbf{2}}+\text { H.c. }
\end{aligned}
$$

This expression has four free parameters: one bilinear and three quartic couplings. The $\mu_{\Delta}$ parameter can be written as a function of the other three parameters by using the scalar potential minimization condition:

$$
\frac{\partial\langle V(\Delta)\rangle}{\partial v_{\Delta}}=16 \kappa_{1} v_{\Delta}^{3}+8 \kappa_{3} v_{\Delta}^{3}-v_{\Delta} \mu_{\Delta}^{2}=0
$$

Solving the leading equation for $\mu_{\Delta}^{2}$ yields the following relation:

$$
\mu_{\Delta}^{2}=8\left(2 \kappa_{1}+\kappa_{3}\right) v_{\Delta}^{2} .
$$

This result indicates that the VEV pattern of the $S_{4}$ doublet $\Delta$ given in Eq. (2.7), is consistent with a global minimum of the scalar potential of Eq. (B1) for a large region of parameter space. The previously described procedure can be used to show that the VEV patterns of the remaining $S_{4}$ doublets of the model are also consistent with the minimization conditions of the scalar potential.

\section{APPENDIX C: THE SCALAR POTENTIAL FOR A $S_{4}$ TRIPLET}

The scalar potential for a $S_{4}$ triplet $S$ has six free parameters: one bilinear and four quartic couplings, as indicated by the relation:

$$
\begin{aligned}
V= & -\mu_{S}^{2}\left(S S^{*}\right)_{\mathbf{1}}+\kappa_{1}\left(S S^{*}\right)_{\mathbf{1}}\left(S S^{*}\right)_{\mathbf{1}}+\kappa_{2}\left(S S^{*}\right)_{\mathbf{3}}\left(S S^{*}\right)_{\mathbf{3}} \\
& +\kappa_{3}\left(S S^{*}\right)_{\mathbf{3}^{\prime}}\left(S S^{*}\right)_{\mathbf{3}^{\prime}}+\kappa_{4}\left(S S^{*}\right)_{\mathbf{2}}\left(S S^{*}\right)_{\mathbf{2}}+\text { H.c. }
\end{aligned}
$$

Its minimization equation allows us to express the $\mu_{S}$ parameter as follows:

$$
\begin{aligned}
\frac{\partial\langle V(S)\rangle}{\partial v_{S}}= & 36 \kappa_{1} v_{S}^{3}+48 \kappa_{2} v_{S}^{3}+4 \kappa_{4}\left(2 e^{\frac{2 i \pi}{3}} v_{S}+2 e^{-\frac{2 i \pi}{3}} v_{S}+2 v_{S}\right) \\
& \times\left(e^{\frac{2 i \pi}{3}} v_{S}^{2}+e^{-\frac{2 i \pi}{3}} v_{S}^{2}+v_{S}^{2}\right)-6 \mu_{S}^{2} v_{S} \\
= & 0 .
\end{aligned}
$$

Here we consider the phase $\omega=e^{2 \pi i / 3}$ arising in the tensor product of $S_{4}$ scalar triplets. Then, we find the following relation for the $\mu_{S}^{2}$ parameter:

$$
\mu_{S}^{2}=2\left(3 \kappa_{1}+4 \kappa_{2}\right) v_{S}^{2} .
$$

This shows that the VEV configuration of the $S_{4}$ triplet $S$ given in Eq. (2.7), is in accordance with the scalar potential minimization condition of Eq. (C2). The remaining $S_{4}$ triplets of the model are also consistent with the minimization conditions of the scalar potential and this can be proved by using the same procedure described before.
[1] H. Georgi and A. Pais, Phys. Rev. D 19, 2746 (1979).

[2] J.W. F. Valle and M. Singer, Phys. Rev. D 28, 540 (1983).

[3] F. Pisano and V. Pleitez, Phys. Rev. D 46, 410 (1992).

[4] R. Foot, O. F. Hernandez, F. Pisano, and V. Pleitez, Phys. Rev. D 47, 4158 (1993).

[5] P. H. Frampton, Phys. Rev. Lett. 69, 2889 (1992).

[6] H. N. Long, Phys. Rev. D 54, 4691 (1996).
[7] H. N. Long, Phys. Rev. D 53, 437 (1996).

[8] R. Foot, H. N. Long, and T. A. Tran, Phys. Rev. D 50, R34 (1994).

[9] A. E. Carcamo Hernandez, R. Martinez, and F. Ochoa, Phys. Rev. D 73, 035007 (2006).

[10] P. V. Dong, H. N. Long, D. V. Soa, and V. V. Vien, Eur. Phys. J. C 71, 1544 (2011).

[11] P. V. Dong, L. T. Hue, H. N. Long, and D. V. Soa, Phys. Rev. D 81, 053004 (2010). 
[12] P. V. Dong, H. N. Long, C. H. Nam, and V. V. Vien, Phys. Rev. D 85, 053001 (2012).

[13] R. H. Benavides, W. A. Ponce, and Y. Giraldo, Phys. Rev. D 82, 013004 (2010).

[14] P. V. Dong, H. N. Long, and H. T. Hung, Phys. Rev. D 86, 033002 (2012).

[15] D. T. Huong, L. T. Hue, M. C. Rodriguez, and H. N. Long, Nucl. Phys. B870, 293 (2013).

[16] P. T. Giang, L. T. Hue, D. T. Huong, and H. N. Long, Nucl. Phys. B864, 85 (2012).

[17] D. T. Binh, L. T. Hue, D. T. Huong, and H. N. Long, Eur. Phys. J. C 74, 2851 (2014).

[18] A. E. Carcamo Hernandez, R. Martinez, and F. Ochoa, Phys. Rev. D 87, 075009 (2013).

[19] A. E. Cárcamo Hernández, R. Martinez, and F. Ochoa, Eur. Phys. J. C 76, 634 (2016).

[20] A. E. Cárcamo Hernández, R. Martinez, and J. Nisperuza, Eur. Phys. J. C 75, 72 (2015).

[21] A. E. Cárcamo Hernández, E. Catańo Mur, and R. Martinez, Phys. Rev. D 90, 073001 (2014).

[22] C. Kelso, H. N. Long, R. Martinez, and F. S. Queiroz, Phys. Rev. D 90, 113011 (2014).

[23] V. V. Vien and H. N. Long, J. High Energy Phys. 04 (2014) 133.

[24] V. Q. Phong, H. N. Long, V. T. Van, and L. H. Minh, Eur. Phys. J. C 75, 342 (2015).

[25] V. Q. Phong, H. N. Long, V. T. Van, and N. C. Thanh, Phys. Rev. D 90, 085019 (2014).

[26] S. M. Boucenna, S. Morisi, and J. W. F. Valle, Phys. Rev. D 90, 013005 (2014).

[27] G. De Conto, A. C. B. Machado, and V. Pleitez, Phys. Rev. D 92, 075031 (2015).

[28] S. M. Boucenna, J. W. F. Valle, and A. Vicente, Phys. Rev. D 92, 053001 (2015).

[29] S. M. Boucenna, S. Morisi, and A. Vicente, Phys. Rev. D 93, 115008 (2016).

[30] R. H. Benavides, L. N. Epele, H. Fanchiotti, C. G. Canal, and W. A. Ponce, Adv. High Energy Phys. 2015, 1 (2015).

[31] A. E. Cárcamo Hernández and R. Martinez, Nucl. Phys. B905, 337 (2016).

[32] L. T. Hue, H. N. Long, T. T. Thuc, and T. P. Nguyen, Nucl. Phys. B907, 37 (2016).

[33] A. E. C. Hernández and I. Nišandžić, Eur. Phys. J. C 76, 380 (2016).

[34] R. M. Fonseca and M. Hirsch, J. High Energy Phys. 08 (2016) 003.

[35] V. V. Vien, A. E. Cárcamo Hernández, and H. N. Long, Nucl. Phys. B913, 792 (2016).

[36] A. E. Cárcamo Hernández, H. N. Long, and V. V. Vien, Eur. Phys. J. C 76, 242 (2016).

[37] R. M. Fonseca and M. Hirsch, Phys. Rev. D 94, 115003 (2016).

[38] F. F. Deppisch, C. Hati, S. Patra, U. Sarkar, and J. W. F. Valle, Phys. Lett. B 762, 432 (2016).

[39] M. Reig, J. W. F. Valle, and C. A. Vaquera-Araujo, Phys. Rev. D 94, 033012 (2016).

[40] A. E. Cárcamo Hernández, S. Kovalenko, H. N. Long, and I. Schmidt, J. High Energy Phys. 07 (2018) 144.

[41] A. E. Cárcamo Hernández and H. N. Long, J. Phys. G 45, 045001 (2018).
[42] C. Hati, S. Patra, M. Reig, J. W. F. Valle, and C. A. Vaquera-Araujo, Phys. Rev. D 96, 015004 (2017).

[43] E. R. Barreto, A. G. Dias, J. Leite, C. C. Nishi, R. L. N. Oliveira, and W. C. Vieira, Phys. Rev. D 97, 055047 (2018).

[44] A. E. Cárcamo Hernández, H. N. Long, and V. V. Vien, Eur. Phys. J. C 78, 804 (2018).

[45] V. V. Vien, H. N. Long, and A. E. Cárcamo Hernández, Mod. Phys. Lett. A 34, 1950005 (2019).

[46] A. G. Dias, J. Leite, D. D. Lopes, and C. C. Nishi, Phys. Rev. D 98, 115017 (2018).

[47] M. M. Ferreira, T. B. de Melo, S. Kovalenko, P. R. D. Pinheiro, and F. S. Queiroz, arXiv:1903.07634.

[48] A. E. Cárcamo Hernández, Y. Hidalgo Velásquez, and N. A. Pérez-Julve, Eur. Phys. J. C 79, 828 (2019).

[49] A. E. Cárcamo Hernández, D. T. Huong, and H. N. Long, arXiv:1910.12877.

[50] C. A. de Sousa Pires and O. P. Ravinez, Phys. Rev. D 58, 035008 (1998); 58, 035008 (1998).

[51] P. V. Dong and H. N. Long, Int. J. Mod. Phys. A 21, 6677 (2006).

[52] J. C. Montero, V. Pleitez, and O. Ravinez, Phys. Rev. D 60, 076003 (1999).

[53] J. C. Montero, C. C. Nishi, V. Pleitez, O. Ravinez, and M. C. Rodriguez, Phys. Rev. D 73, 016003 (2006).

[54] P. B. Pal, Phys. Rev. D 52, 1659 (1995).

[55] A. G. Dias, V. Pleitez, and M. D. Tonasse, Phys. Rev. D 67 , 095008 (2003).

[56] A. G. Dias and V. Pleitez, Phys. Rev. D 69, 077702 (2004).

[57] A. G. Dias, C. A. de S. Pires, and P. S. Rodrigues da Silva, Phys. Rev. D 68, 115009 (2003).

[58] J. K. Mizukoshi, C. A. de S.Pires, F. S. Queiroz, and P. S. Rodrigues da Silva, Phys. Rev. D 83, 065024 (2011).

[59] A. G. Dias, C. A. de S.Pires, and P. S. Rodrigues da Silva, Phys. Rev. D 82, 035013 (2010).

[60] J. D. Ruiz-Alvarez, C. A. de S. Pires, F. S. Queiroz, D. Restrepo, and P. S. Rodrigues da Silva, Phys. Rev. D 86, 075011 (2012).

[61] D. Cogollo, A. X. Gonzalez-Morales, F. S. Queiroz, and P. R. Teles, J. Cosmol. Astropart. Phys. 1411 (2014) 002.

[62] P. S. Rodrigues da Silva, Phys. Int. 7, 15 (2016).

[63] G. Altarelli, F. Feruglio, and L. Merlo, J. High Energy Phys. 05 (2009) 020.

[64] F. Bazzocchi, L. Merlo, and S. Morisi, Phys. Rev. D 80, 053003 (2009).

[65] F. Bazzocchi, L. Merlo, and S. Morisi, Nucl. Phys. B816, 204 (2009).

[66] R. de Adelhart Toorop, F. Bazzocchi, and L. Merlo, J. High Energy Phys. 08 (2010) 001.

[67] K. M. Patel, Phys. Lett. B 695, 225 (2011).

[68] S. Morisi, K. M. Patel, and E. Peinado, Phys. Rev. D 84, 053002 (2011).

[69] G. Altarelli, F. Feruglio, L. Merlo, and E. Stamou, J. High Energy Phys. 08 (2012) 021.

[70] R. N. Mohapatra and C. C. Nishi, Phys. Rev. D 86, 073007 (2012).

[71] P. S. Bhupal Dev, B. Dutta, R. N. Mohapatra, and M. Severson, Phys. Rev. D 86, 035002 (2012).

[72] I. de Medeiros Varzielas and L. Lavoura, J. Phys. G 40, 085002 (2013). 
[73] G. J. Ding, S. F. King, C. Luhn, and A. J. Stuart, J. High Energy Phys. 05 (2013) 084.

[74] H. Ishimori, Y. Shimizu, M. Tanimoto, and A. Watanabe, Phys. Rev. D 83, 033004 (2011).

[75] G. J. Ding and Y. L. Zhou, Nucl. Phys. B876, 418 (2013).

[76] C. Hagedorn and M. Serone, J. High Energy Phys. 10 (2011) 083.

[77] M. D. Campos, A. E. Cárcamo Hernández, H. Päs, and E. Schumacher, Phys. Rev. D 91, 116011 (2015).

[78] V. V. Vien, H. N. Long, and D. P. Khoi, Int. J. Mod. Phys. A 30, 1550102 (2015).

[79] F. J. de Anda, S. F. King, and E. Perdomo, J. High Energy Phys. 12 (2017) 075; 04 (2019) 69.

[80] F. J. de Anda and S. F. King, J. High Energy Phys. 07 (2018) 057.

[81] A. E. Cárcamo Hernández and S.F. King, arXiv:1903. 02565.

[82] P. T. Chen, G. J. Ding, S. F. King, and C. C. Li, arXiv: 1906.11414.

[83] I. De Medeiros Varzielas, S. F. King, and Y. L. Zhou, arXiv:1906.02208.

[84] I. De Medeiros Varzielas, M. Levy, and Y. L. Zhou, Phys. Rev. D 100, 035027 (2019).

[85] C. D. Froggatt and H. B. Nielsen, Nucl. Phys. B147, 277 (1979).

[86] R. N. Mohapatra and J. W. F. Valle, Phys. Rev. D 34, 1642 (1986).

[87] E. K. Akhmedov, M. Lindner, E. Schnapka, and J. W. F. Valle, Phys. Lett. B 368, 270 (1996).

[88] E. K. Akhmedov, M. Lindner, E. Schnapka, and J. W. F. Valle, Phys. Rev. D 53, 2752 (1996).

[89] M. Malinsky, J. C. Romao, and J. W. F. Valle, Phys. Rev. Lett. 95, 161801 (2005).

[90] D. Borah and B. Karmakar, Phys. Lett. B 789, 59 (2019).

[91] M. Hirsch, S. Morisi, and J. W. F. Valle, Phys. Lett. B 679, 454 (2009).

[92] C. O. Dib, G. R. Moreno, and N. A. Neill, Phys. Rev. D 90, 113003 (2014).

[93] M. Chakraborty, H. Z. Devi, and A. Ghosal, Phys. Lett. B 741, 210 (2015).

[94] R. Sinha, R. Samanta, and A. Ghosal, Phys. Lett. B 759, 206 (2016).

[95] C. Salazar, R. H. Benavides, W. A. Ponce, and E. Rojas, J. High Energy Phys. 07 (2015) 096.

[96] V. T. N. Huyen, H. N. Long, T. T. Lam, and V. Q. Phong, Commun. Phys. 24, 97 (2014).

[97] R. Martinez and F. Ochoa, Phys. Rev. D 77, 065012 (2008).

[98] A. J. Buras, F. De Fazio, and J. Girrbach, J. High Energy Phys. 02 (2014) 112.

[99] A. J. Buras, F. De Fazio, and J. Girrbach-Noe, J. High Energy Phys. 08 (2014) 039.

[100] A. J. Buras, F. De Fazio, J. Girrbach, and M. V. Carlucci, J. High Energy Phys. 02 (2013) 023.

[101] R. A. Diaz, R. Martinez, and F. Ochoa, Phys. Rev. D 69, 095009 (2004).

[102] H. N. Long, N. V. Hop, L. T. Hue, N. H. Thao, and A. E. Cárcamo Hernández, Phys. Rev. D 100, 015004 (2019).

[103] M. A. Perez, G. Tavares-Velasco, and J. J. Toscano, Phys. Rev. D 69, 115004 (2004).
[104] M. Aaboud et al. (ATLAS Collaboration), J. High Energy Phys. 01 (2018) 055.

[105] K. Bora, Horizons in biochemistry and biophysics 2, 112 (2013).

[106] Z.z. Xing, H. Zhang, and S. Zhou, Phys. Rev. D 77, 113016 (2008).

[107] C. Patrignani et al. (Particle Data Group), Chin. Phys. C 40, 100001 (2016).

[108] P. S. B. Dev and R. N. Mohapatra, Phys. Rev. D 81, 013001 (2010).

[109] P. S. Bhupal Dev, R. Franceschini, and R. N. Mohapatra, Phys. Rev. D 86, 035002 (2012).

[110] A. Das and N. Okada, Phys. Rev. D 88, 113001 (2013).

[111] C. H. Lee, P. S. Bhupal Dev, and R. N. Mohapatra, Phys. Rev. D 88, 093010 (2013).

[112] A. Das, P. S. Bhupal Dev, and N. Okada, Phys. Lett. B 735, 364 (2014).

[113] A. Das, P. Konar, and S. Majhi, J. High Energy Phys. 06 (2016) 019.

[114] A. Das, P. Konar, and A. Thalapillil, J. High Energy Phys. 02 (2018) 083.

[115] A. Das and N. Okada, Phys. Lett. B 774, 32 (2017).

[116] A. Das, P. S. B. Dev, and C.S. Kim, Phys. Rev. D 95, 115013 (2017).

[117] A. Das, Y. Gao, and T. Kamon, Eur. Phys. J. C 79, 424 (2019).

[118] A. Das, S. Jana, S. Mandal, and S. Nandi, Phys. Rev. D 99, 055030 (2019).

[119] A. Das, Adv. High Energy Phys. 2018, 9785318 (2018).

[120] A. Bhardwaj, A. Das, P. Konar, and A. Thalapillil, arXiv: 1801.00797.

[121] J. C. Helo, H. Li, N. A. Neill, M. Ramsey-Musolf, and J. C. Vasquez, Phys. Rev. D 99, 055042 (2019).

[122] S. Pascoli, R. Ruiz, and C. Weiland, J. High Energy Phys. 06 (2019) 049.

[123] P. I. Krastev and S. T. Petcov, Phys. Lett. B 205, 84 (1988).

[124] P. F. de Salas, D. V. Forero, C. A. Ternes, M. Tortola, and J. W. F. Valle, Phys. Lett. B 782, 633 (2018).

[125] M. A. Shifman, A. I. Vainshtein, M. B. Voloshin, and V. I. Zakharov, Yad. Fiz. 30, 1368 (1979) [Sov. J. Nucl. Phys. 30, 711 (1979)].

[126] M. B. Gavela, G. Girardi, C. Malleville, and P. Sorba, Nucl. Phys. B193, 257 (1981).

[127] P. Kalyniak, R. Bates, and J. N. Ng, Phys. Rev. D 33, 755 (1986).

[128] J. F. Gunion, H. E. Haber, G. L. Kane, and S. Dawson, Front. Phys. 80, 1 (2000).

[129] M. Spira, Fortschr. Phys. 46, 203 (1998).

[130] A. Djouadi, Phys. Rep. 459, 1 (2008).

[131] W. J. Marciano, C. Zhang, and S. Willenbrock, Phys. Rev. D 85, 013002 (2012).

[132] L. Wang and X.F. Han, Phys. Rev. D 86, 095007 (2012).

[133] A. E. Carcamo Hernandez, C. O. Dib, and A. R. Zerwekh, Eur. Phys. J. C 74, 2822 (2014).

[134] G. Bhattacharyya and D. Das, Phys. Rev. D 91, 015005 (2015). 
[135] E. C. F. S. Fortes, A. C. B. Machado, J. Montaño, and V. Pleitez, J. Phys. G 42, 115001 (2015).

[136] A. E. Carcamo Hernandez, C. O. Dib, and A. R. Zerwekh, Nucl. Part. Phys. Proc. 267-269, 35 (2015).

[137] A. E. Cárcamo Hernández, I. de Medeiros Varzielas, and E. Schumacher, Phys. Rev. D 93, 016003 (2016).

[138] A. E. Cárcamo Hernández, B. Díaz Sáez, C. O. Dib, and A. Zerwekh, Phys. Rev. D 96, 115027 (2017).
[139] V. Khachatryan et al. (CMS Collaboration), Eur. Phys. J. C 74, 3076 (2014).

[140] G. Aad et al. (ATLAS Collaboration), Phys. Rev. D 90, 112015 (2014).

[141] H. Ishimori, T. Kobayashi, H. Ohki, Y. Shimizu, H. Okada, and M. Tanimoto, Prog. Theor. Phys. Suppl. 183, 1 (2010). 